\title{
Characterization of a $\mathrm{C3}$ deoxygenation pathway reveals a key branch point in aminoglycoside biosynthesis
}

Meinan Lv, Xinjian Ji, Junfeng Zhao, Yongzhen Li, Chen Zhang, Li Su, Wei Ding, Zixin

Deng, Yi Yu and Qi Zhang

\section{Supplementary Methods}

\section{Instrumentation}

High-performance liquid chromatography (HPLC) was performed using a Thermo Scientific Dionex Ultimate 3000 system with an evaporative light scattering detector (Alltech, 2000ES) equipped with a DIKMA Diamonsil C18 column $(3.5 \mu \mathrm{m}, 150 \times 2.1 \mathrm{~mm})$. High resolution mass spectra (HRMS) were acquired using a Q-Exactive ${ }^{\mathrm{TM}}$ Focus Hybrid Quadrupole-Orbitrap Mass Spectrometer (Thermo Fisher Scientific Inc.) equipped with a Dionex Ultimate 3000 HPLC system (Thermo Fisher), or an LTQ XL Orbitrap Mass Spectrometer equipped with an Accela 600 U-HPLC system (Thermo Fisher Scientific Inc.), or a Synapt ESI quadrupole ToF Mass Spectrometry System (Waters) equipped with an Acquity Ultra Performance Liquid Chromatography (UPLC) system (Waters). NMR spectra were recorded using Varian Inova 500 MHz NMR spectrometer. UV-vis spectroscopy analysis was performed on a 1900 double beam UV-vis spectrometer (Yoke Instrument Co. Ltd., Shanghai, China). EPR spectra were obtained at X-band using a Bruker EMX plus 10/12 spectrometer system, equipped with an Oxford ESR910 liquid helium continuous flow cryostat (Oxford Instrument Co.). PCR was performed on a Bio-Rad T100 ${ }^{\mathrm{TM}}$ Thermal Cycler using Phanta Max Super-Fidelity DNA Polymerase (Vazyme Biotech) or KOD DNA polymerase (TOYOBO).

\section{Chemicals and biochemicals}

All chemical reagents and anhydrous solvents were purchased from commercial sources and used without further purification unless otherwise specified. S-adenosyl-L-methionine (SAM), thiostrepton, paromycin, neomycin, tobramycin, and spectinomycin were purchased from Sangon 
Biotech Co. Ltd (Shanghai, China). Lividomycin was purchased from Biolise Co. Ltd (Xiamen, China). $\mathrm{Fe}\left(\mathrm{NH}_{4}\right)_{2}\left(\mathrm{SO}_{4}\right)_{2} \bullet 6 \mathrm{H}_{2} \mathrm{O}$ and $\mathrm{Na}_{2} \mathrm{~S}$ were from Sigma-Aldrich Co. Ltd (USA). Kanamycin and culture media were from Sinopharm Chemical Reagent Co. Ltd (China). Gentimicin X2, gentimicin G418, and the GenQ expressing plasmid pET28/VM_GenQ are precious gifts from Prof. Yuhui Sun (Wuhan University). ${ }^{1}$ Enzymes were from Takara Biotechnology (Dalian, China) or from Vazyme Biotech (Nanjing, China) unless otherwise specified. Primers were synthesized at Invitrogen Biotech Co. Ltd (Shanghai, China).

\section{DNA sequencing and analysis}

S. tenebrarius genomic DNA was prepared using UltraClean Microbial DNA Isolation Kit (Anbiosci Ltd, Shenzhen, China), and was then sequenced by Illumina Hiseq 2000 with a 300 bp paired-end library through TruSeq method at GenScript Co. Ltd (Nanjing, China). A total of 3,685,798 paired-reads were obtained and assembled by using the assembly programs $\mathrm{SOAPdeNOVO}^{2}$ with the parameters "sequence length $>25 \mathrm{bp}$ and base quality $>20$ ". When the K-mer was 21 , the best assembly result could be obtained. The resultant 384 contigs were further annotated by RAST (http://rast.nmpdr.org/rast.cgi).

\section{Construction of the plasmids for genetic manipulation of Streptomyces tenebrarius}

Because the apramycin producer Streptomyces tenebrarius is resistant to apramycin, in this study we constructed two plasmids containing a spectinomycin resistance gene aadA for genetic manipulation of $S$. tenebrarius. aadA was amplified by PCR using a primer pair aadA-F and aadA-R (Supplementary Table 2), and the plasmid pIJ778 ${ }^{3}$ as the template. The $\sim 1.1 \mathrm{~kb}$ DNA fragment containing aadA and its promoter region was digested by SacI, and recovered using a Qiagen PCR purification kit. This DNA fragment was ligated with a $\sim 2.7 \mathrm{~kb}$ fragment obtained by SacI digestion of pOJ260, ${ }^{4}$ or a $~ 5.2 \mathrm{~kb}$ fragment obtained by SacI digestion of pIB139, using T4 DNA ligase (Takara Biotechnology). Chemically competent E. coli DH5 $\alpha$ cells were transformed with the ligation mixture and plated on LB-agar containing spectinomycin $(50 \mu \mathrm{g} / \mathrm{mL})$ 
to screen for positive clones. Clones were confirmed by DNA sequencing at Genewiz Co. Ltd (Suzhou, China). The resulting plasmids pWHU258 (pOJ260 derivative) and pWHU268 (pIB139 derivative) were used for gene-knockout and complementation experiments, respectively.

\section{Construction of the $\triangle a p r D 4$ mutant}

To inactivate aprD4, a 2241 bp upstream fragment and 2400 bp downstream fragment were amplified separately from the $S$. tenebrarius genomic DNA by PCR using the primer pair aprD4-Left-F and aprD4-Left-R, and the pair aprD4-Right-F and aprD4-Right-R, respectively (Supplementary Table 2). The resulting fragments were purified using a Qiagen PCR purification kit, and then cloned into the HindIII/EcoRI site of pWHU258 using In-fusion HD Cloning Kit (Clontech) to give the in-frame deletion construct pWHU288. pWHU288 was then transferred into S. tenebrarius via E. coli-Streptomyces conjugation. The aprD4 in-frame deletion mutant (designated as WDY288) was screened and its genotype was confirmed by PCR and DNA sequencing, and by Southern blot (Figure S1).

\section{Construction of the $\operatorname{AaprD3}$ mutant}

To inactivate $a p r D 3$, a 2265 bp upstream fragment and 2289 bp downstream fragment were amplified separately from the $S$. tenebrarius genomic DNA by PCR using the primer pair aprD3-Left-F and aprD3-Left-R, and the pair aprD3-Right-F and aprD3-Right-R, respectively (Supplementary Table 2). The resulting fragments were purified using a Qiagen PCR purification kit, and then cloned into the HindIII/EcoRI site of pWHU258 using In-fusion HD Cloning Kit (Clontech) to give the in-frame deletion construct pWHU289. pWHU289 was then transferred into

S. tenebrarius via E. coli-Streptomyces conjugation. The aprD3 in-frame deletion mutant (designated as WDY289) was screened and its genotype was confirmed by PCR and DNA sequencing, and by Southern blot (Figure S12)

\section{Construction of the $\operatorname{\Delta aprQ}$ mutant}


To inactivate aprQ, a $2217 \mathrm{bp}$ upstream fragment and $2232 \mathrm{bp}$ downstream fragment were amplified separately from the S. tenebrarius genomic DNA by PCR using the primer pair aprQ-Left-F and aprQ-Left-R, and the pair aprQ-Right-F and aprQ-Right-R, respectively (Supplementary Table 2). The resulting fragments were purified using a Qiagen PCR purification kit, and then cloned into the HindIII/EcoRI site of pWHU258 using In-fusion HD Cloning Kit (Clontech) to give the in-frame deletion construct pWHU290. pWHU290 was then transferred into $S$. tenebrarius via E. coli-Streptomyces conjugation. The aprQ in-frame deletion mutant (designated as WDY290) was screened and its genotype was confirmed by PCR and DNA sequencing, and by Southern blot (Figure S20)

\section{Construction of the $\Delta t o b Q$ mutant}

To inactivate tobQ, a $2213 \mathrm{bp}$ upstream fragment and $2135 \mathrm{bp}$ downstream fragment were amplified separately from the S. tenebrarius genomic DNA by PCR using the primer pair tobQ-Left-F and tobQ-Left-R, and the pair tobQ-Right-F and tobQ-Right-R, respectively (Supplementary Table 2). The resulting fragments were purified using a Qiagen PCR purification kit, and then cloned into the HindIII/EcoRI site of pWHU258 using In-fusion HD Cloning Kit (Clontech) to give the in-frame deletion construct pWHU291. Plasmid pWHU291 was then transferred into S. tenebrarius via E. coli-Streptomyces conjugation. The tobQ in-frame deletion mutant (designated as WDY291) was screened and its genotype was confirmed by PCR and DNA sequencing.

\section{Construction of the $\triangle a p r Q \Delta t o b Q$ double knockout mutant}

To obtain $\triangle a p r Q \Delta t o b Q$ double in-frame deletion mutant, the tobQ in-frame deletion construct pWHU191 was transferred into the $\triangle a p r Q$ mutant WDY290 via E. coli-Streptomyces conjugation. The $\triangle a p r Q \Delta t o b Q$ double in-frame deletion mutant (designated as WDY292) was screened and its genotype was confirmed by PCR and DNA sequencing, and by Southern blot (Figure S21). 


\section{Construction of the $\triangle a p r D 4 \Delta a p r Q$ mutant}

To obtain $\triangle a p r D 4 \Delta a p r Q$ double in-frame deletion mutant, a 2248 bp upstream fragment and a $2400 \mathrm{bp}$ downstream fragment were amplified separately from the $\triangle a p r Q$ mutant WDY290 genomic DNA by PCR using the primer pair aprD4aprQ-F and aprD4-Left-R, and the pair aprD4-Right-F and aprD4-Right-R, respectively (Supplementary Table 2). The resulting fragments were purified using a Qiagen PCR purification kit, and then cloned into the HindIII/EcoRI site of pWHU258 using In-fusion HD Cloning Kit (Clontech) to give the in-frame deletion construct pWHU293. Plasmid pWHU293 was then transferred into the $\triangle a p r Q$ mutant WDY290 via $E$. coli-Streptomyces conjugation. The $\triangle a p r D 4 \Delta a p r Q$ double in-frame deletion mutant (designated as WDY293) was screened and its genotype was confirmed by PCR and DNA sequencing, and by Southern blot (Figure S23).

\section{Construction of the $\triangle a p r D 4 \Delta t o b Q$ mutant}

To obtain $\triangle a p r D 4 \Delta t o b Q$ double in-frame deletion mutant, the tobQ in-frame deletion construct pWHU291 was then transferred into the $\triangle a p r D 4$ mutant WDY288 via E. coli-Streptomyces conjugation. The $\triangle a p r D 4 \Delta t o b Q$ double in-frame deletion mutant (designated as WDY294) was screened and its genotype was confirmed by PCR and DNA sequencing, and by Southern blot (Figure S22).

\section{Southern blot analysis}

Genomic DNAs of S. tenebrarius wild type and mutant strains were prepared according to the standard protocol ${ }^{6}$. DNA samples were digested with appropriate restriction enzymes, separated by electrophoresis on agarose gels, and blotted on Hybond- $\mathrm{N}^{+}$positive-charged nylon membranes (Roche). Hybridization was carried out according to the manufacturer's instruction (Roche) by using probes labeled with digoxigenin-11-dUTP (Roche).

\section{Complementation of the gene-knockout mutants}


The complementation plasmids were prepared by cloning aprD4 (PCR using a primer pair HB-aprD4-F and HB-aprD4-R), aprD3 (PCR using a primer pair HB-aprD3-F and HB-aprD3-R), AprQ (PCR using a primer pair HB-aprQ-F and HB-aprQ-R), and tobQ (PCR using a primer pair HB-tobQ-F and HB-tobQ-R) into the NdeI/EcoRI site of pWHU268 to result in pWHU300, pWHU301, pWH302, pWHU303 for complementation of aprD4, aprD3, aprQ, tobQ, respectively

(Figure S 2-3). These pWHU268 derivative plasmids, which can be integrated into the attB site in Streptomyces chromosome via phage $\Phi \mathrm{C} 31$ integrase-catalyzed site-specific recombination, ${ }^{7,8}$ were each transferred into a gene knockout mutant via E. coli-Streptomyces conjugation to give WDY300 ( $\triangle a p r D 4$ complementation by aprD4), WDY301 ( $\triangle a p r D 3$ complementation by aprD3), WDY302 ( $\triangle a p r Q$ complementation by aprQ), WDY303 ( $\Delta$ tobQ complementation by tobQ), WDY306 ( $\triangle a p r Q \Delta t o b Q$ complementation by aprQ), WDY307 ( $\triangle a p r D 4 \Delta a p r Q$ complementation by aprQ), and WDY308 ( $\triangle a p r D 4 \Delta a p r Q$ complementation by tobQ).

\section{In vivo production of aminoglycosides and their biosynthetic intermediates}

SPA medium (in grams per liter of solution: soluble starch, 20; beaf extract, $1 ; \mathrm{MgSO}_{4}, 0.5 ; \mathrm{KNO}_{3}$, $1 ; \mathrm{NaCl}, 0.5 ; \mathrm{K}_{2} \mathrm{HPO}_{4}, 0.5 ;$ agar, 20) was used for spore production. The spore suspension of $S$. tenebrarius and the related mutants were inoculated into 5ml TSBY medium (in grams per liter of solution: trypticase soy broth, $30.0 \mathrm{~g}$; yeast extract, 3.0; $\mathrm{NaCl} 13.0 \mathrm{~g} ; \mathrm{MgCl}_{2} \bullet 6 \mathrm{H}_{2} \mathrm{O}, 4.0 \mathrm{~g}$; $\mathrm{MgSO}_{4} \cdot 7 \mathrm{H} 2 \mathrm{O}, 3.45 \mathrm{~g} ; \mathrm{KCl}, 0.34 \mathrm{~g} ; \mathrm{CaCl}_{2} \cdot 2 \mathrm{H}_{2} \mathrm{O}, 0.14 \mathrm{~g}$; the $\mathrm{pH}$ was adjusted to 7.2 before autoclave at $120^{\circ} \mathrm{C}$ for $15 \mathrm{~min}$ ). The culture was grown at $37^{\circ} \mathrm{C}$ and $220 \mathrm{rpm}$ for $36 \mathrm{~h}$ and was used to inoculate $50 \mathrm{ml}$ SPC fermentation medium containing the followings (in grams per liter of solution): glucose, 40; peptone, 10; soybean meal, 4; corn meal, $10 ; \mathrm{MgSO}_{4} \cdot 7 \mathrm{H}_{2} \mathrm{O}, 4 ; \mathrm{NH}_{4} \mathrm{Cl}, 5$; $\mathrm{FeSO}_{4}, 0.5 ; \mathrm{MnCl}_{2}, 0.3 ; \mathrm{ZnSO}_{4}, 0.03 ; \mathrm{CaCO}_{3}, 5$. The cells were grown at $37^{\circ} \mathrm{C}$ and $220 \mathrm{rpm}$ for 7 days, and the culture supernatant was collected by centrifugation at $5000 \mathrm{rpm}$ for $15 \mathrm{~min}$, and was adjusted to a $\mathrm{pH}$ of $2 \sim 3$ with oxalate. After removal of the insoluble fraction by centrifugation at $5000 \mathrm{rpm}$ for $30 \mathrm{~min}$, the solution was passed through a column containing $5 \mathrm{ml} 732$ cation exchange resin (Hebi Juxing Resinco., Ltd, Hebi, China). The column was then washed with 50 $\mathrm{mL}$ of distilled water followed by $10 \mathrm{~mL} \mathrm{3 \%}$ ammonia hydroxide solution. The eluate was purged 
with a nitrogen stream and then taken to dryness by lyophilization. The residue was dissolved in $500 \mu \mathrm{L}$ of methanol before HPLC and LCMS analysis.

\section{Isolation and structure characterization of oxyapramycin, lividamine, and paromamine}

Oxyapramycin and paromamine were isolated from the fermentation culture of the $\triangle a p r D 4$ mutant WDY288, and lividamine was isolated from the fermentation culture of the $\triangle a p r Q$ mutant WDY290. The culture supernatant was adjusted to a $\mathrm{pH}$ of $2 \sim 3$ with oxalate and the insoluble fraction was removed by centrifugation ( $5000 \mathrm{rpm}, 30 \mathrm{~min}$ ). The supernatant was extracted by the crude 732 cation exchange resin. The resulting resin was packed on a column, washed by distilled water and then by $3 \%$ ammonia hydroxide solution. The ammonia hydroxide eluate was then passed through an 801 anion-exchange column, and the eluate was purged with a nitrogen stream and then taken to dryness by lyophilization. The lyophilized residue was then separated by semi-preparative HPLC using a DIONEX System equipped with UltiMate 3000 Pump connecting with Evaporative Light Scattering Detector (Alltech, 2000ES) with an Agilent ZORBAX SB-C18 column $(5 \mu \mathrm{m}, 9.4 \times 250 \mathrm{~mm})$. The column was equilibrated with $80 \%$ solvent $\mathrm{A}\left(\mathrm{H}_{2} \mathrm{O}, 10 \mathrm{mM}\right.$ heptafluorobutyric acid) and $20 \%$ solvent $\mathrm{B}\left(\mathrm{CH}_{3} \mathrm{CN}\right)$, and developed at a flow rate of $3 \mathrm{ml} / \mathrm{min}$ : 0-3 min, constant $80 \% \mathrm{~A} / 20 \% \mathrm{~B}$; 3-5 min, a linear gradient to $75 \% \mathrm{~A} / 25 \% \mathrm{~B}$; $5-9$ min, a linear gradient to $71 \% \mathrm{~A} / 29 \% \mathrm{~B}$; $9-15 \mathrm{~min}$, a linear gradient to $65 \% \mathrm{~A} / 35 \% \mathrm{~B}, 15-20 \mathrm{~min}$, a linear gradient to $62 \% \mathrm{~A} / 38 \% \mathrm{~B} ; 20-30 \mathrm{~min}$, a linear gradient to $80 \% \mathrm{~A} / 20 \% \mathrm{~B}$.

\section{Construction of plasmids for overexpressing N-terminally hexa-His-tagged AprD3 and}

\section{AprD4}

The aprD3 and aprD4 genes were amplified from the S. tenebrarius genomic DNA using a primer pair N-AprD3-F and N-AprD3-R, and a pair N-AprD4-F and N-AprD4-R (Table S1), respectively. The PCR amplified products were digested with NcoI and XhoI, purified using a Qiagen PCR purification kit, and inserted into the same restriction site of the expression vector pET28a (Novagen). Chemically competent $E$. coli $\mathrm{DH} 5 \alpha$ cells were transformed with the ligation mixture 
and plated on LB-agar containing kanamycin $(50 \mu \mathrm{g} \mathrm{mL}-1)$ to screen for positive clones, which were confirmed by DNA sequencing.

\section{Construction of the plasmid for overexpressing N-terminally Flag-tagged AprD3}

The aprD3 gene was amplified from the $S$. tenebrarius genomic DNA using a primer pair N-flag-AprD3-F and N-AprD3-R (table S2) carrying an engineered Flag tag. The PCR amplified products were digested with NcoI and XhoI, purified using a Qiagen PCR purification kit, and inserted into the same restriction site of the expression vector pET28a (Novagen). Chemically competent $E$. coli DH5 $\alpha$ cells were transformed with the ligation mixture and plated on LB-agar containing kanamycin $(50 \mu \mathrm{g} / \mathrm{mL})$ to screen for positive clones, which were confirmed by DNA sequencing.

\section{AprD4 mutagenesis}

To reveal the $[4 \mathrm{Fe}-4 \mathrm{~S}]$ cluster binding site of AprD4, all 12 Cys residues in AprD4 were individually replaced by an Ala residue, and this was achieved by using a site-directed mutagenesis procedure reported previously. ${ }^{9}$ PCR was performed with each primer pair (e.g. AprD4-C203A-F and AprD4-C203A-R for producing AprD4 C203A, etc., the primer sequences are listed in Supplementary Table 2), and the AprD4-expressing plasmid as the template. After checking the PCR products by agarose gel electrophoresis, $1 \mu \mathrm{L}$ of the PCR product was treated with the restriction enzyme DpnI (TOYOBO), and the total $20 \mu \mathrm{L}$ reaction solution was incubated at $37{ }^{\circ} \mathrm{C}$ for $\sim 1.5$ h. $2.5 \mu \mathrm{L}$ of the resulting solution was used for transformation of $E$. coli DH10B cells. The positive clones were picked and verified by DNA sequencing.

\section{Protein Expression}

E. coli BL21 (DE3) cells were transformed via electroporation with the expression plasmids. A single colony transformant was used to inoculate a $5 \mathrm{~mL}$ culture of LB supplemented with 100 $\mu \mathrm{g} / \mathrm{mL}$ kanamycin. The culture was grown at $37{ }^{\circ} \mathrm{C}$ for $12 \mathrm{~h}$ and was used to inoculate $1 \mathrm{~L}$ of LB 
medium containing $100 \mu \mathrm{g} / \mathrm{mL}$ kanamycin. Cells were grown at $37^{\circ} \mathrm{C}$ and $220 \mathrm{rpm}$ to an $\mathrm{OD}_{600} \sim$ 0.6-0.8, and then IPTG was added to a final concentration of $0.1 \mathrm{mM}$. To express AprD4 wild type and mutant enzymes, sterilized $\mathrm{Fe}\left(\mathrm{NH}_{4}\right)_{2}\left(\mathrm{SO}_{4}\right)_{2}$ solution was added to the culture to a final concentration of $10 \mu \mathrm{M}$. After additional $8 \mathrm{~h}$ of incubation, the cells were harvested by centrifugation at $5000 \mathrm{rpm}$ for $15 \mathrm{~min}$ at $4{ }^{\circ} \mathrm{C}$. The pellet was used directly for protein purification or stored at $-80{ }^{\circ} \mathrm{C}$ before use.

\section{Purification of AprD3 and GenQ}

AprD3 and GenQ were purified aerobically. The cell pellet collected by centrifugation was re-suspended in $20 \mathrm{ml}$ lysis buffer (50 mM MOPS, $200 \mathrm{mM} \mathrm{NaCl}$, and 10\% glycerol, $\mathrm{pH} 8.0$ ), and was lysed by a high pressure homogenizer (FB-110X, Shanghai Litu Mechanical Equipment Engineering Co., Ltd, China). Cell debris was removed via centrifugation at $14000 \mathrm{rpm}$ for $30 \mathrm{~min}$ at $4{ }^{\circ} \mathrm{C}$. The supernatant was incubated with $4 \mathrm{ml} \mathrm{Ni-NTA}$ resin pre-equilibrated with the lysis buffer, and then subjected to affinity purification on a column. The desired elution fractions were combined and concentrated using an Amicon Ultra-15 Centrifugal Filter Unit, and the concentrated protein solution was desalted using a DG-10 column (Bio-Rad) pre-equilibrated with the elution buffer (50 mM MOPS, $25 \mathrm{mM} \mathrm{NaCl}$, and 10\% (v/v) glycerol, $\mathrm{pH} 8.0$ ). The protein fraction was collected and concentrated, analyzed by SDS-PAGE (10\% Tris-glycine gel), and was used directly for in vitro assay or stored at $-80{ }^{\circ} \mathrm{C}$ upon further use. Protein concentration was determined using a Bradford assay kit (Promega) using bovine serum albumin (BSA) as a standard.

\section{Preparation of the reconstituted AprD4 wild type and mutant enzymes}

Purification and [4Fe-4S] reconstitution of AprD4 wild type and mutant enzymes were performed in an anaerobic glove box (Coy Laboratory Product Inc., USA) with less than $2 \mathrm{ppm}$ of $\mathrm{O}_{2}$. The cell pellet was resuspended in $20 \mathrm{ml}$ of the lysis buffer (50 mM MOPS, $200 \mathrm{mM} \mathrm{NaCl}$, and $10 \%$ glycerol, $\mathrm{pH}$ 8.0), and was lysed by sonication on ice. Cell debris was removed via centrifugation

at $14000 \mathrm{rpm}$ for $30 \mathrm{~min}$ at $4{ }^{\circ} \mathrm{C}$. The supernatant was incubated with $4 \mathrm{ml} \mathrm{Ni-NTA}$ resin pre-equilibrated with the lysis buffer, and then subjected to affinity purification on a column. The 
desired fractions were combined and concentrated using an Amicon Ultra-15 Centrifugal Filter Unit, and analyzed by SDS-PAGE (12\% Tris-glycine gel). Protein concentration was determined using a Bradford assay kit (Promega) using BSA as a standard.

\section{Native-PAGE analysis}

Native-PAGE analysis of AprD4 and AprD3 mixtures, which were incubated anaerobically at room temperature for $30 \mathrm{~min}$ or $2 \mathrm{~h}$, was carried out with a 12\% Tris-glycine gel. SDS was omitted in the analysis and the electrophoresis was performed at $4{ }^{\circ} \mathrm{C}$.

\section{Western blot}

SDS-PAGE analysis was run in a $12 \%$ gel for $\sim 2 \mathrm{~h}$ at $120 \mathrm{~V}$. Proteins were transferred from the gel to a PVDF membrane; the latter was then cut into two parts from a line roughly around $40 \mathrm{kDa}$, allowing separation of AprD3 and AprD4 into two PVDF membranes. The membranes were incubated in a PBS blocking buffer containing $5 \%$ skim milk at $4{ }^{\circ} \mathrm{C}$ overnight, and were washed twice with PBST buffer $\left(\mathrm{NaCl} 8 \mathrm{~g} / \mathrm{L}, \mathrm{KCl} 0.2 \mathrm{~g} / \mathrm{L}, \mathrm{Na}_{2} \mathrm{HPO}_{4} \bullet 12 \mathrm{H}_{2} \mathrm{O}, 3.58 \mathrm{~g} / \mathrm{L} \mathrm{KH}_{2} \mathrm{PO}_{4}, 0.24 \mathrm{~g} / \mathrm{L}\right.$, $0.05 \%$ tween). 1:2500 anti-Flag tag or anti-His tag mouse monocolonal antibodies were used to probe AprD3 and AprD4, respectively, and proteins were visualized by chemiluminescence by using 1:5000 IPKine HRP (Abbkine) and goat anti-mouse IgG fc secondary antibody (Abbkine, for AprD3) or goat anti-mouse igG LCS antibody (Abbkine, for AprD4).

\section{Construction of pWDYHS01 for expressing proteins in Streptomyces}

A $179 \mathrm{bp}$ fragment containing an $\mathrm{N}$-terminal $\mathrm{His}_{6}{ }_{6}$-tag and multiple cloning sites were amplified from pET28a by PCR using the primer pair pGM1190_F and pGM1190_R (Supplementary Table 2). PCR was carried out in $20 \mu \mathrm{L}$ of volume with 5\% DMSO and KOD DNA polymerase (TOYOBO), and the resulting fragments were cloned into $\mathrm{pGM} 1190^{10}$ by using an In-fusion HD Cloning Kit (Clontech) to yield the plasmid pWDYHS01 for protein expression in Streptomyces.

\section{Construction of the aprQ-expressing Streptomyces strain WDY320}


The gene aprQ was amplified by high fidelity PCR from $S$. tenebrarius genomic DNA using oligonucleotide primer pair N-AprQ-F and N-AprQ-R (Supplementary Table 2) carrying the engineered NdeI and XhoI restriction sites. The PCR-amplified gene product was purified, digested with NdeI and XhoI, and ligated into the NdeI/XhoI cleaved expression vector pWDYHS01 to generate pWHU320. pWHU320 was then transferred into Streptomyces lividans TK24. The resulting colonies were selected by aac(3)IV resistance marker and confirmed by PCR to obtain the recombinant strain WDY320.

\section{Construction of the tobQ-expressing Streptomyces strain WDY321}

tob $Q$ was amplified by high fidelity PCR from $S$. tenebrarius genomic DNA using oligonucleotide primer pair N-TobQ-F and N-TobQ-R (Supplementary Table 2) carrying the engineered NdeI and XhoI restriction sites. The PCR-amplified product was purified, digested with NdeI and XhoI, and ligated into the NdeI/XhoI cleaved expression vector pWDYHS01 to yield pWHU321. pWHU321 was then transferred into Streptomyces lividance TK24, and the resulting colonies were selected by $a a c(3) I V$ resistance marker and confirmed by PCR to obtain the recombinant strain WDY321.

\section{Phylogenetic analysis}

The protein sequences in this study were obtained from the GenBank database; their accession numbers and the source organisms are listed in Supplementary Table 1. The sequences were aligned in Clustal $\mathrm{X}^{11}$ using default parameters with iteration at each alignment step, and the alignments were manually fine-tuned afterwards. Bayesian inference was used to calculate posterior probability of clades utilizing the program MrBayes (version 3.2). ${ }^{12}$ Final analyses consisted of two sets of eight chains each (one cold and seven heated), run for about 1 million generations with trees saved and parameters sampled every 100 generations. Analyses were run to reach a convergence with standard deviation of split frequencies $<0.008$. Posterior probabilities 
were averaged over the final $75 \%$ of trees ( $25 \%$ burn in). The analysis utilized a mixed amino acid model with a proportion of sites designated invariant, and rate variation among sites modeled after a gamma distribution divided into eight categories, with all variable parameters estimated by the program based on BioNJ starting trees. The figures of the Bayesian phylograms were prepared by using Tree-View. ${ }^{13}$ 
Figure S1. In-frame deletion of aprD4 in S. tenebrarius, showing the schematic representation of the in-frame deletion and the southern blot analysis. The probe is shown as a purple bar, and the arrows beside the southern blot data indicate the size of the expected signal fragments.
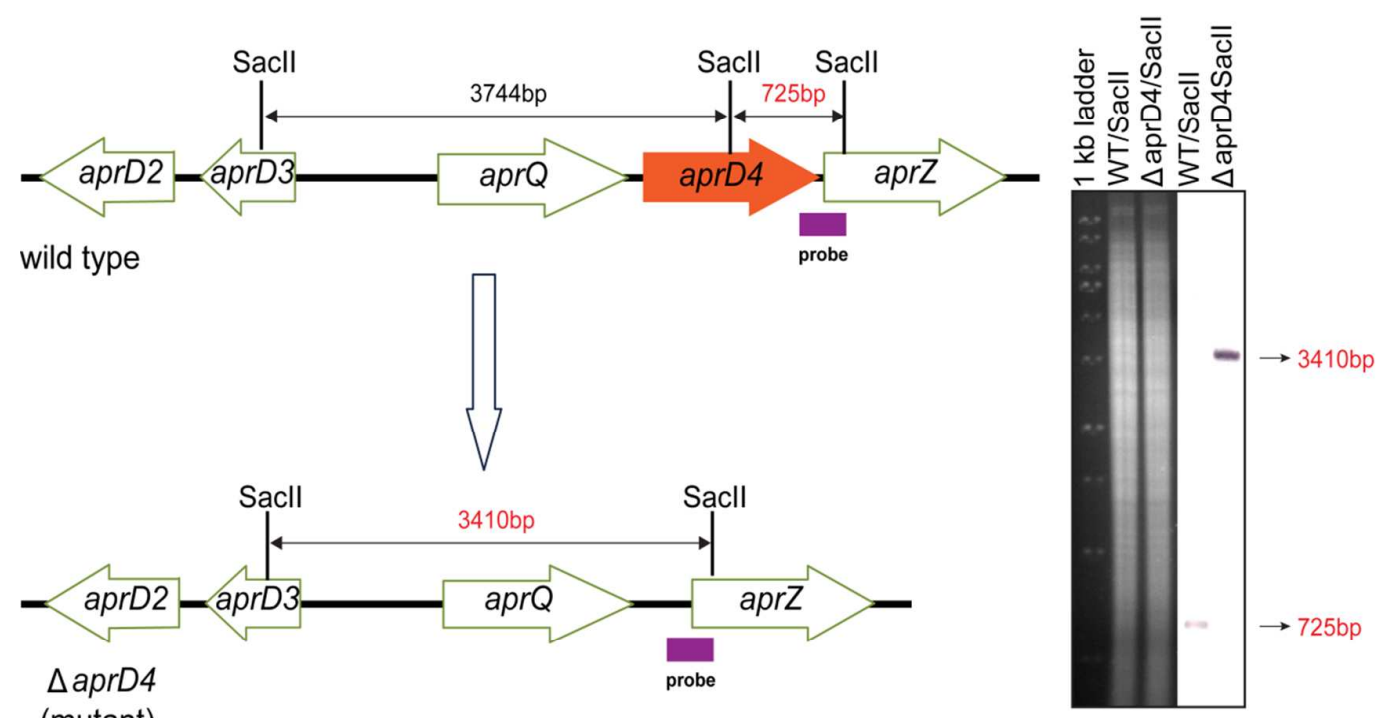

(mutant) 
Figure S2. HR-MS/MS analysis of oxyapramycin purified from the fermentation culture of the saprD4 mutant, showing (A) the collision induced dissociation (CID) fragments, and (B) the MS/MS spectrum.

A
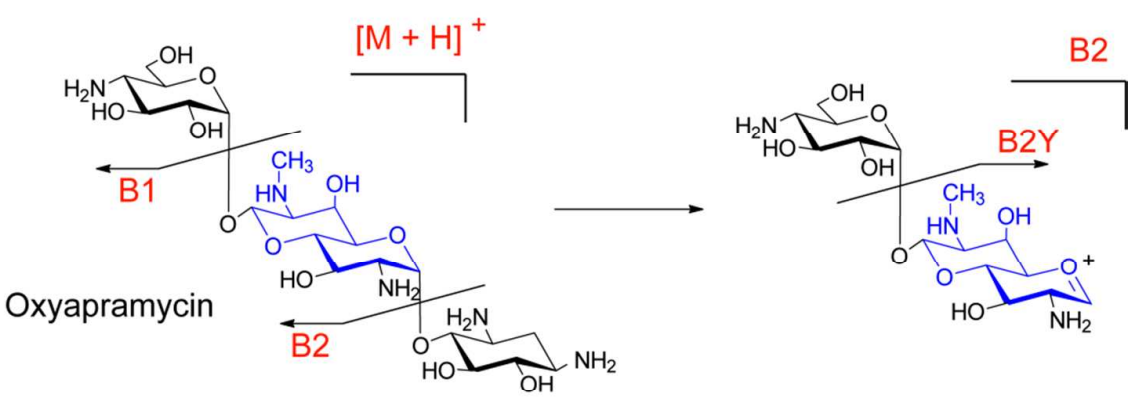

B

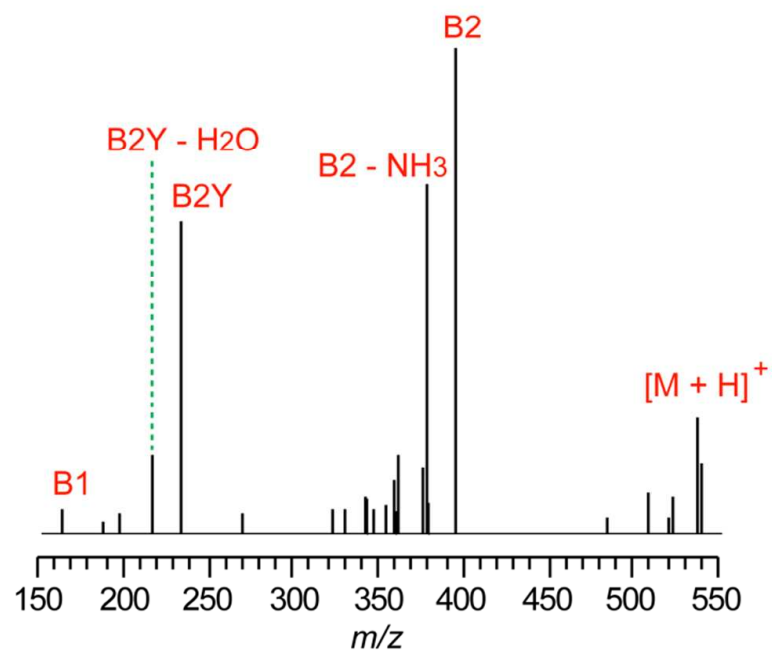


Figure S3. ${ }^{1} \mathrm{H}$ NMR analysis of oxyapramycin purified from the fermentation culture of the $\triangle a p r D 4$ mutant, showing (A) the chemical structure of oxyapramycin, and (B) the ${ }^{1} \mathrm{H}$ NMR spectrum.
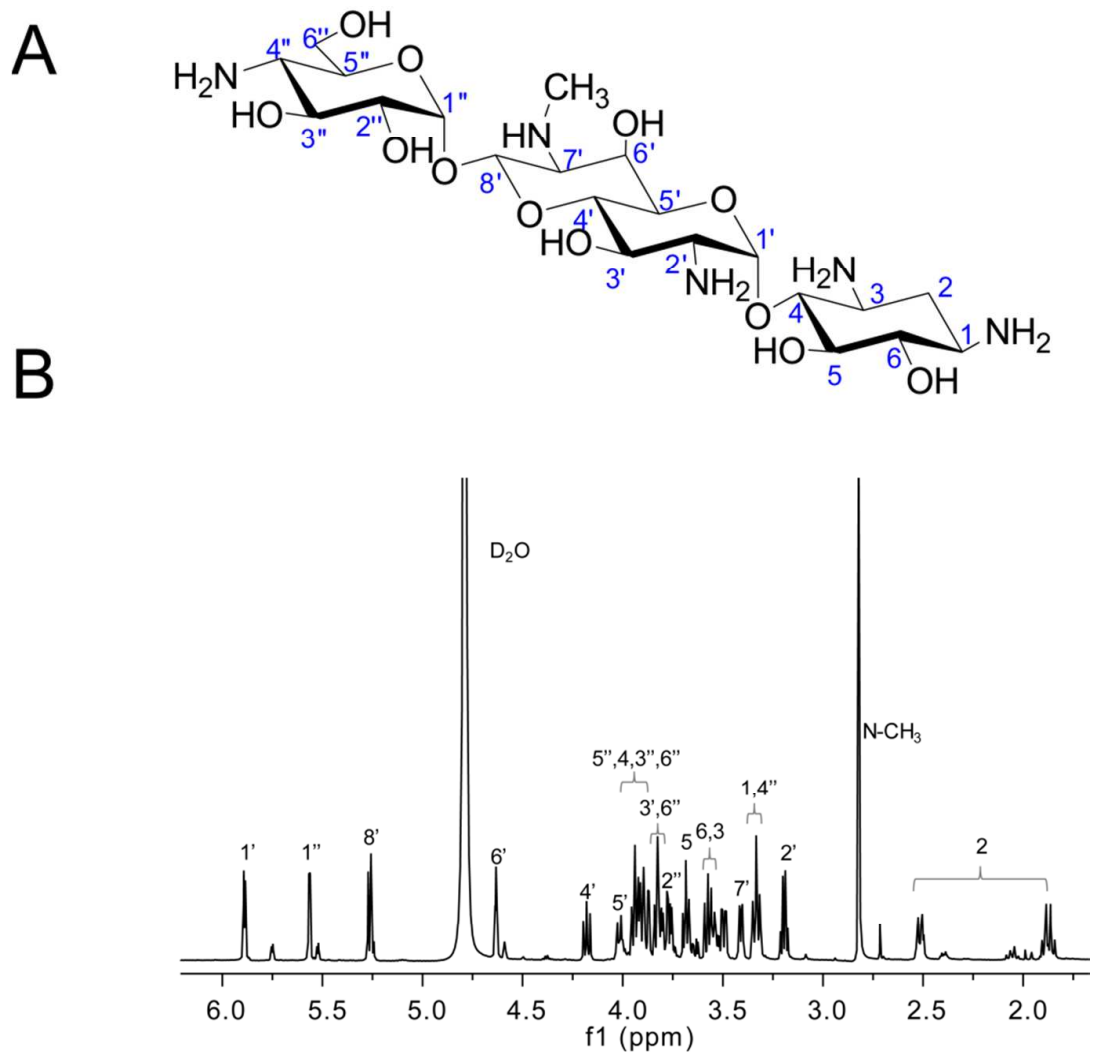
Figure S4. ${ }^{13} \mathrm{C}$ NMR analysis of oxyapramycin purified from the fermentation culture of the $\triangle a p r D 4$ mutant, showing (A) the chemical structure of oxyapramycin, and (B) the ${ }^{13} \mathrm{C}$ NMR spectrum.

A

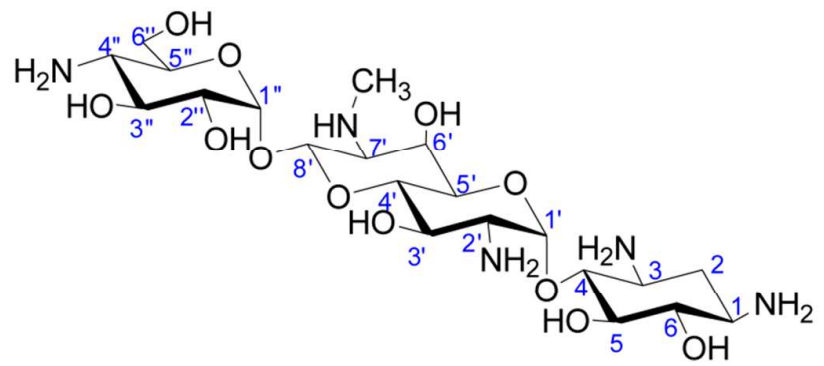

B

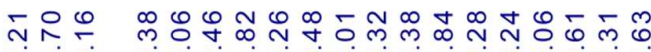

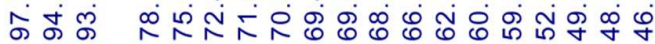

11)

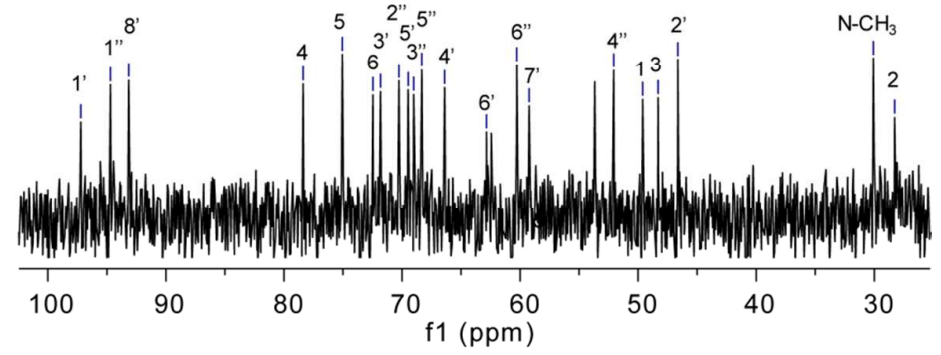


Figure S5. HR-MS/MS analysis of paromamine purified from the fermentation culture of the aaprD4 mutant, showing (A) the collision induced dissociation (CID) fragments, and (B) the MS/MS spectrum.

A

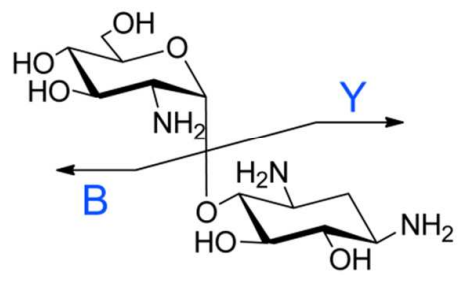

B

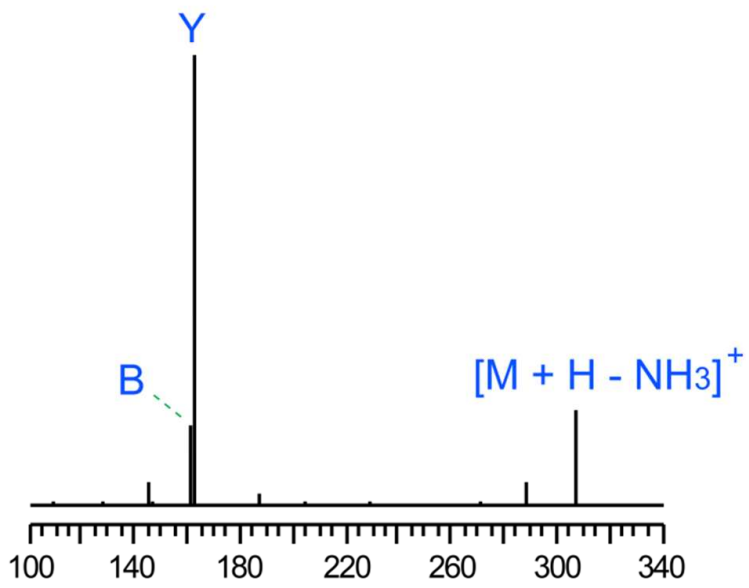


Figure S6. ${ }^{1} \mathrm{H}$ NMR analysis of paromamine purified from the fermentation culture of the $\triangle$ aprD4 mutant, showing (A) the chemical structure of paromamine, and (B) the ${ }^{1} \mathrm{H}$ NMR spectrum.
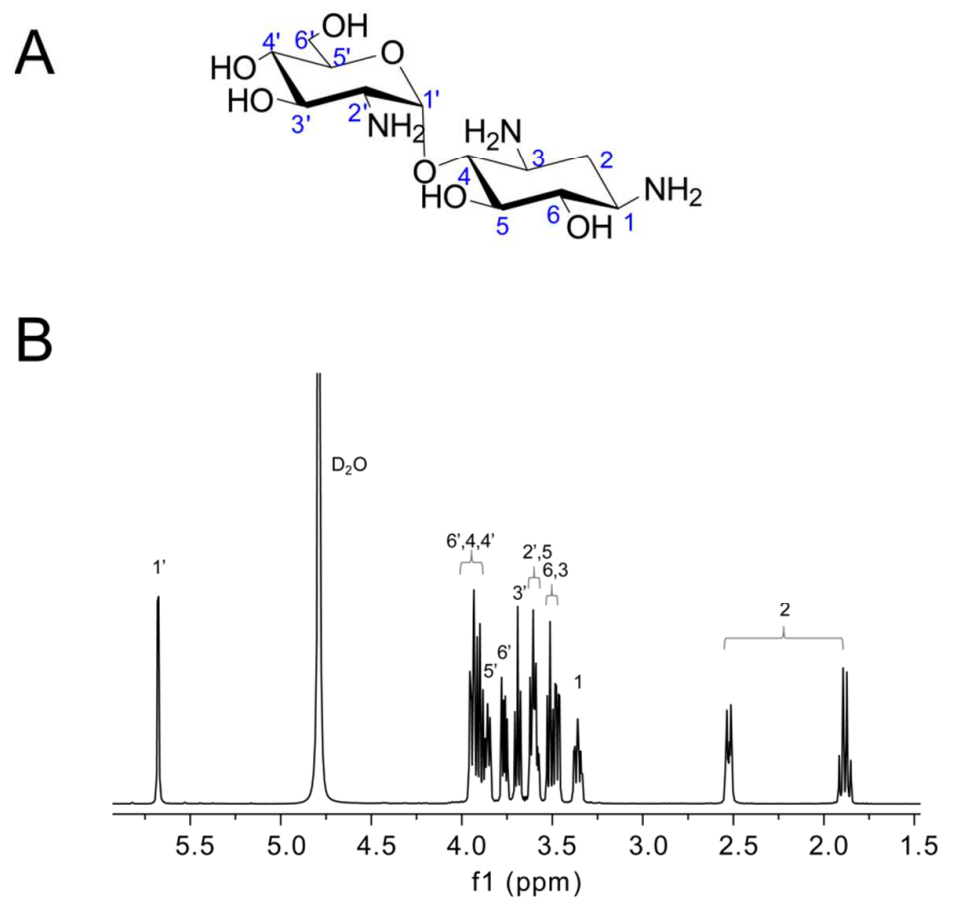
Figure S7. ${ }^{13} \mathrm{C}$ NMR analysis of paromamine purified from the fermentation culture of the $\triangle a p r D 4$ mutant, showing (A) the chemical structure of paromamine, and (B) the ${ }^{13} \mathrm{C} N M R$ spectrum.

A

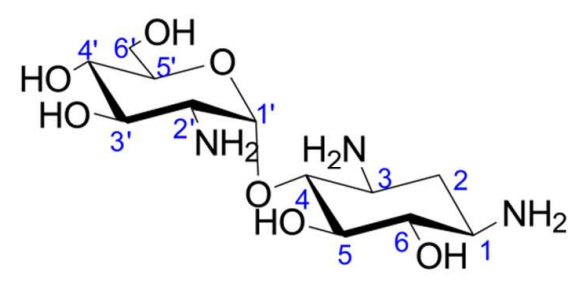

B

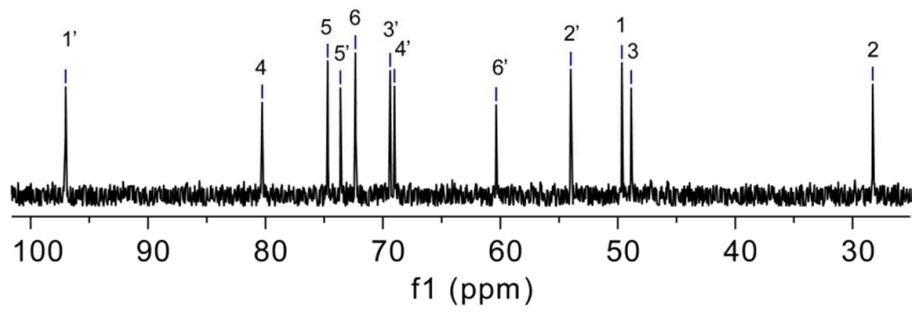


Figure S8. HPLC analysis of the SAM cleavage activity of the 12 AprD4 Cys-to-Ala mutants. The assays were performed by incubating $500 \mu \mathrm{M}$ paromamine with $\sim 40 \mu \mathrm{M}$ reconstituted AprD4 mutant, $1 \mathrm{mM}$ SAM and $4 \mathrm{mM}$ sodium dithionite in $50 \mathrm{mM}$ MOPS buffer $(\mathrm{pH} 8.0)$ at room temperature for $\sim 3 \mathrm{~h}$. Mutants with an intact $\mathrm{CX}_{3} \mathrm{CX}_{3} \mathrm{C}(\mathbf{C P Y P C R F Y C})$ motif all cleaved SAM with varied efficiencies, whereas the three mutants in which the $\mathrm{CX}_{3} \mathrm{CX}_{3} \mathrm{C}(\mathbf{C P Y P C R F Y C})$ motif was altered (shown in red bold font) had no detectable SAM cleavage activity. UV detector, 260 nm.
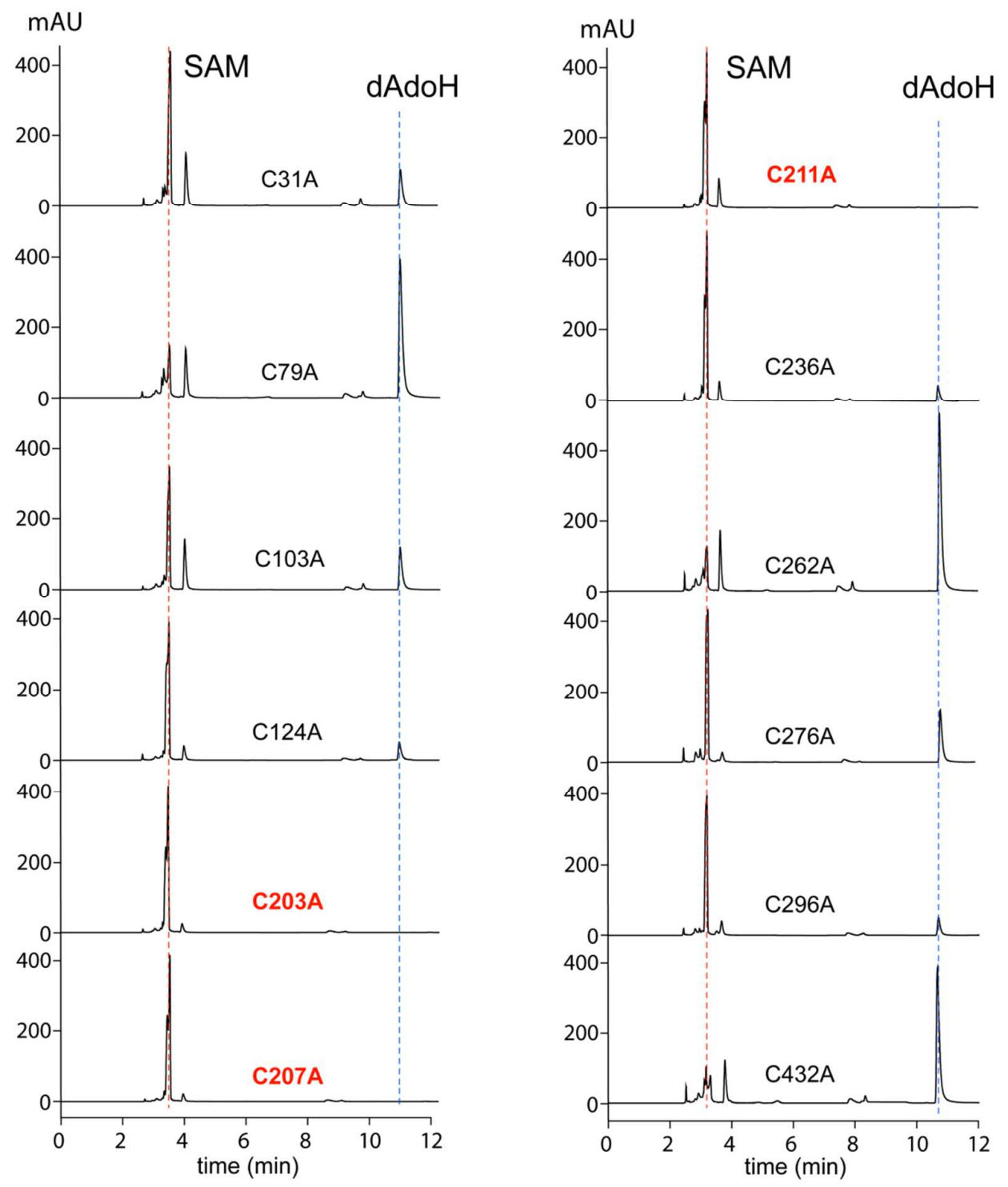
Figure S9. UV-vis spectra of the reconstituted AprD4 C207A mutant (red solid line) and the protein reduced with DTH (blue dashed line).

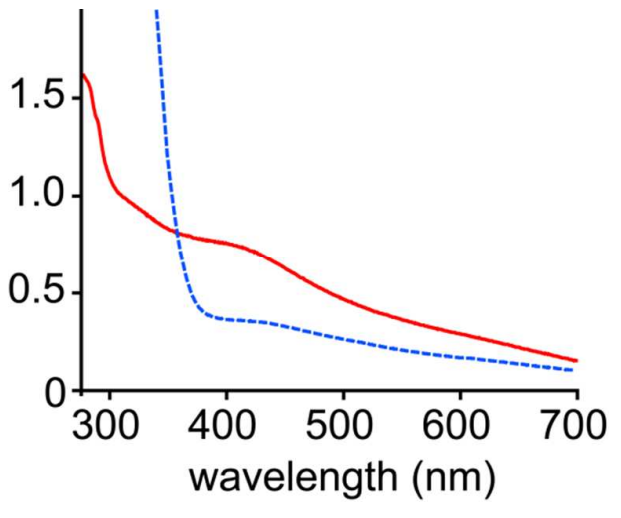


Figure S10. HR-MS/MS spectrum of DOP (4) and its ion fragments upon collision induced dissociation (CID).

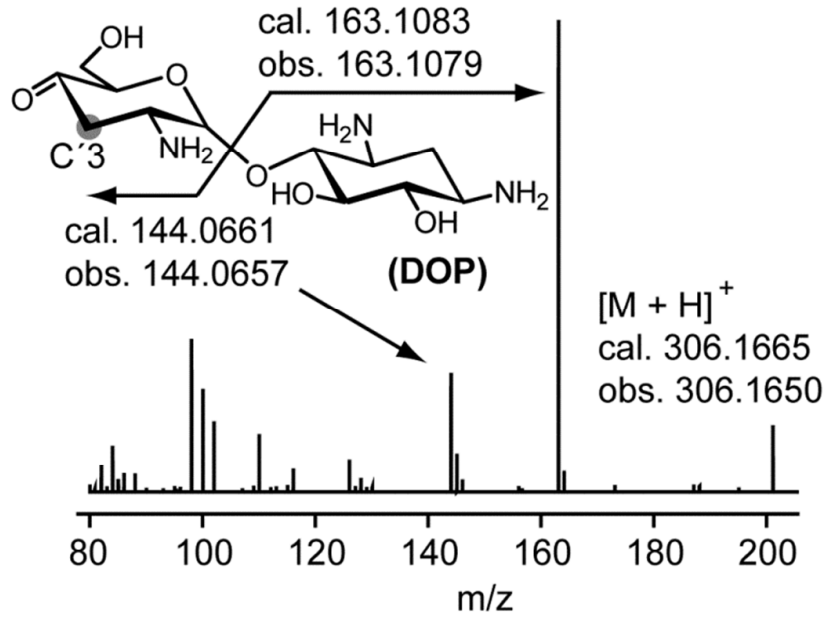


Figure S11. MS analysis of (A) dAdoH and (B) paromamine from AprD4-catalyzed reaction, which was performed in a buffer containing $67 \% \mathrm{D}_{2} \mathrm{O}$ and $100 \mu \mathrm{M}$ paromamine, and incubated at room temperature for $5 \mathrm{~h}$. Significant deuterium incorporation into paromamine was observed but deuterium incorporation into dAdoH was not apparent. (C) A hypothetic model for AprD4-catalzyed deuterium incorporation into paromamine. The substrate radical could abstract a deuterium atom from an unidentified source (e.g. a protein residue) or be reduced by DTH coupled with a deuterium transfer. Notably, similar observations of substrate deuteriation were also found in the study of the radical SAM enzymes DesII ${ }^{14,15}$ and NosL $\mathrm{L}^{16}$.

A

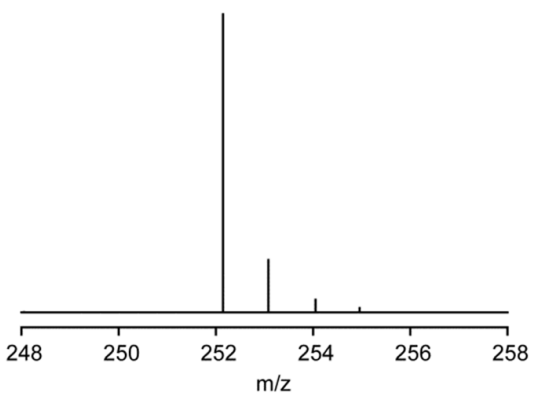

B

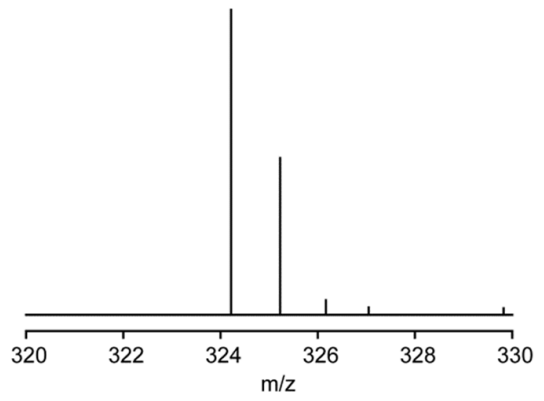

C

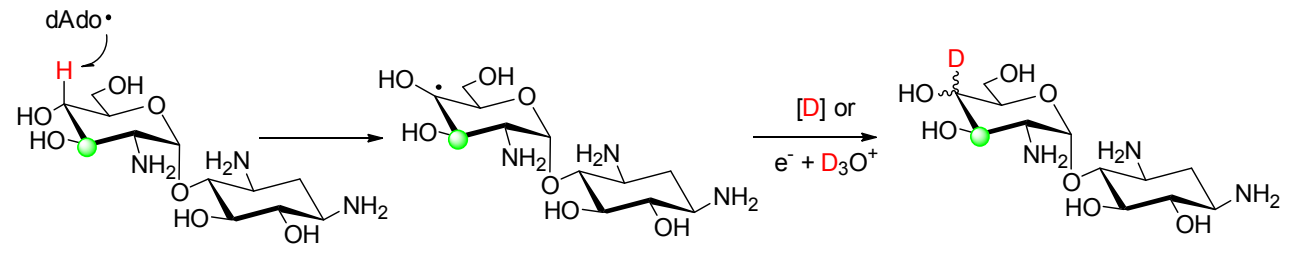


Figure S12. In-frame deletion of aprD3 in S. tenebrarius, showing the schematic representation of the in-frame deletion and the southern blot analysis. The probe is shown as a purple bar, and the arrows beside the southern blot data indicate the size of the expected signal fragments.

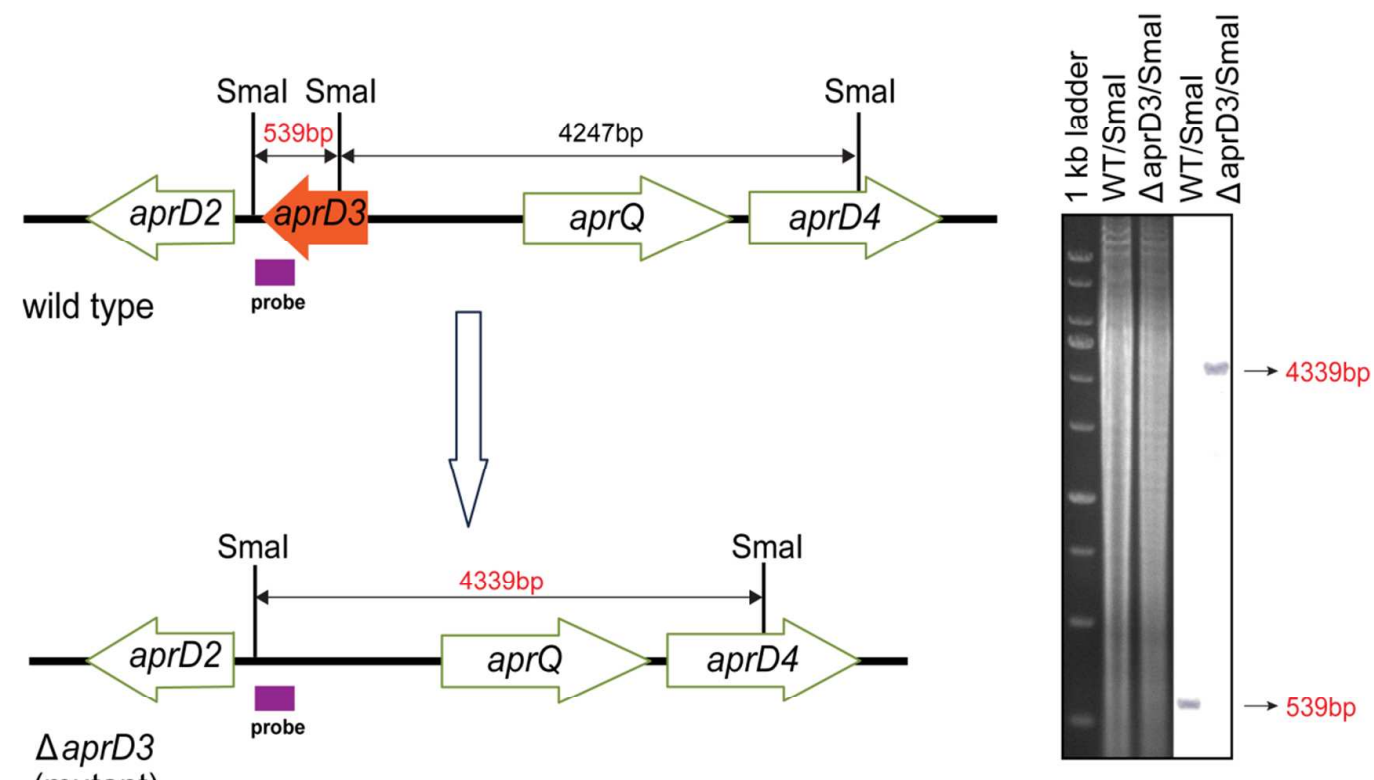


Figure S13. Structural confirmation of lividamine by using comparative MS analysis. (A) EICs of $[\mathrm{M}+\mathrm{H}]^{+}=308.2$ (corresponding to lividamine) for (i) reaction with AprD4 and AprD3 using paromamine as the substrate, and (ii) lividamine purified from the $\triangle a p r Q$ mutant (also see Figure S 14-16). (B) MS/MS spectra of (i) the compound with $[\mathrm{M}+\mathrm{H}]^{+}=308.2$ from the tandem reaction catalyzed by AprD4 and AprD3, and (ii) purified lividamine standard.

A
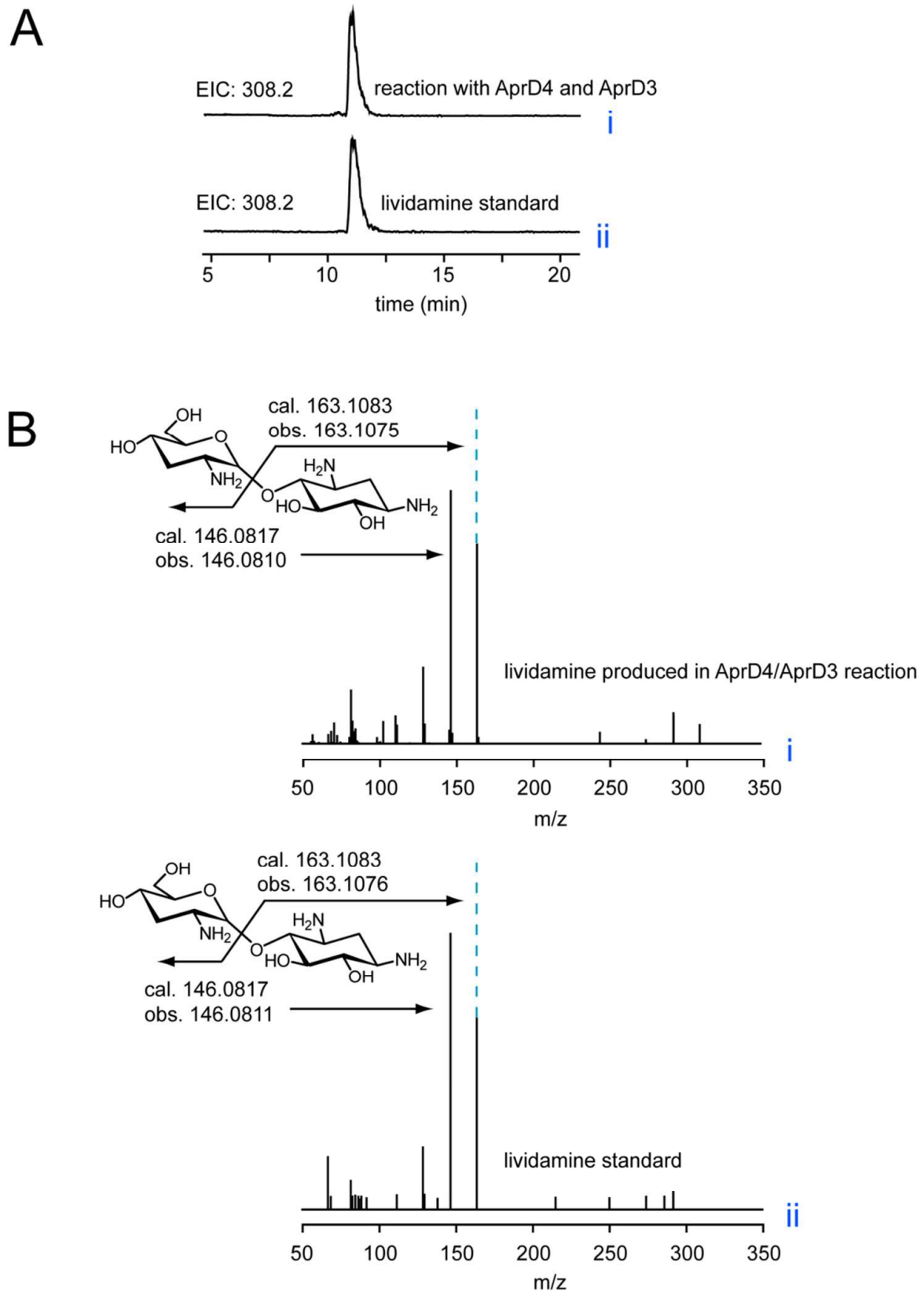
Figure S14. HR-MS/MS analysis of lividamine purified from the fermentation culture of the $\triangle a p r Q$ mutant, showing (A) the collision induced dissociation (CID) fragments, and (B) the MS/MS spectrum.

A

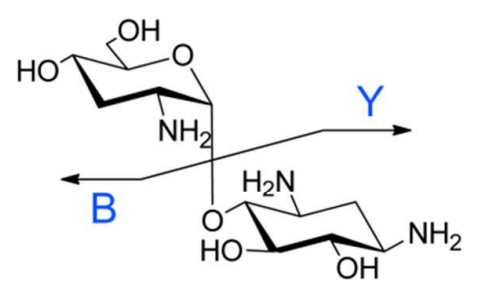

B

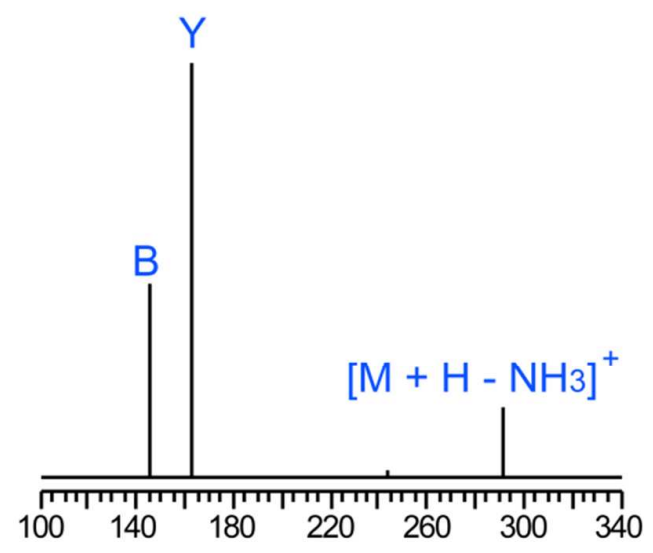


Figure S15. ${ }^{1} \mathrm{H}$ NMR analysis of lividamine purified from the fermentation culture of the $\triangle a p r Q$ mutant, showing (A) the chemical structure of lividamine, and (B) the ${ }^{1} \mathrm{H}$ NMR spectrum.

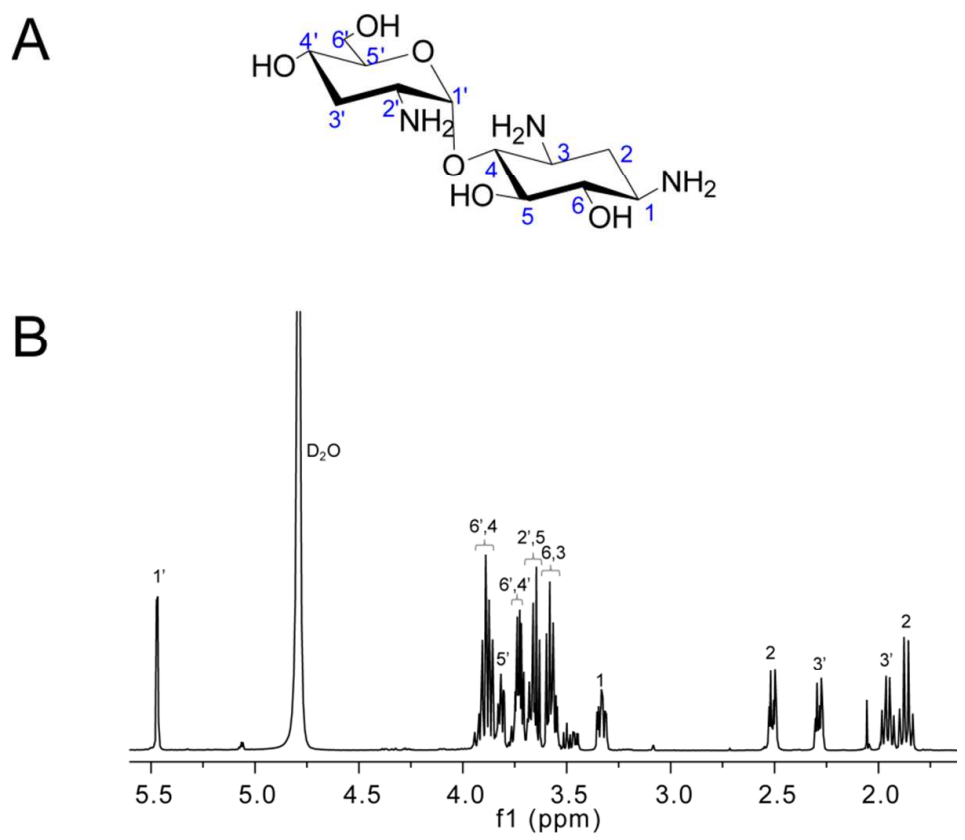


Figure S16. ${ }^{13} \mathrm{C}$ NMR analysis of lividamine purified from the fermentation culture of the $\triangle a p r Q$ mutant, showing (A) the chemical structure of lividamine, and (B) the ${ }^{13} \mathrm{C}$ NMR spectrum.

A

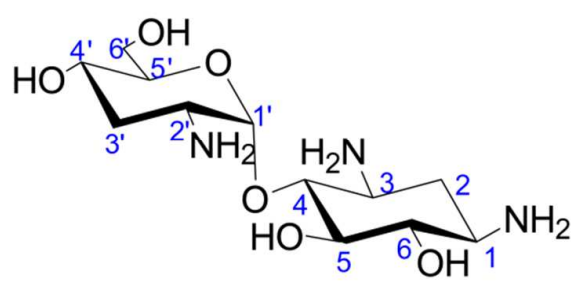

B

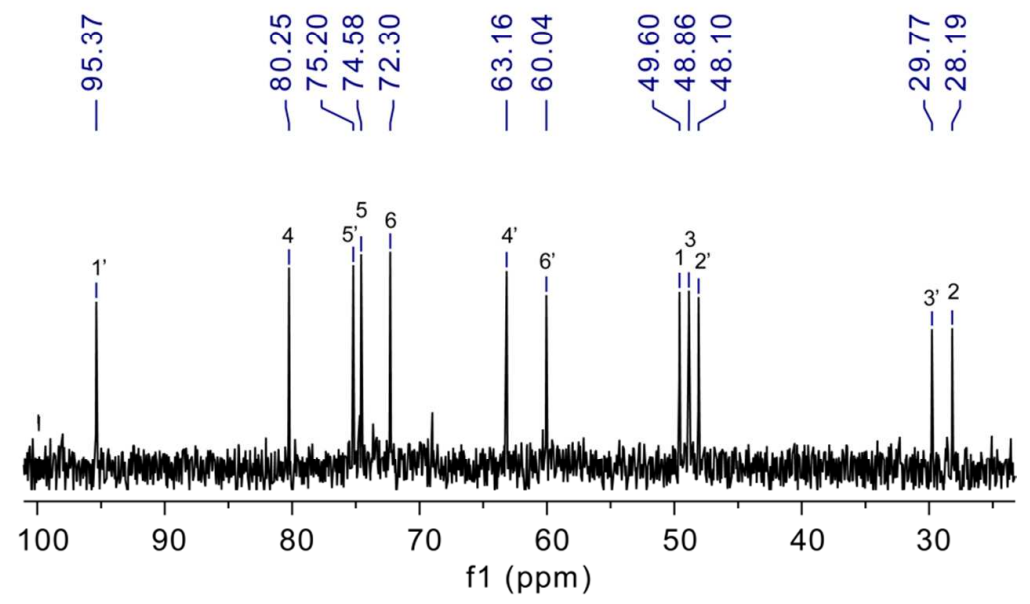


Figure S17. C3 deoxygenation involved in ascarylose biosynthesis, which is catalyzed by the coupled E1/E3 enzyme system. E1 and E3 form a catalytic complex and were co-eluted in a FPLC gel-filtration analysis. ${ }^{17}$ Both dehydration and reduction occur at the E1 active site.

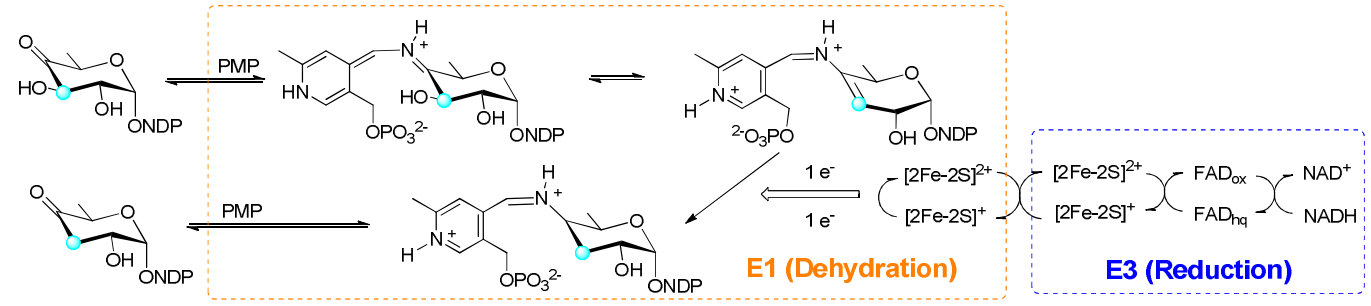


Figure S18. AprD4 and AprD3 do not form a tight protein complex. (A) SDS-PAGE analysis of purified AprD3 and AprD4. (B) Native PAGE analysis of AprD4 and AprD3 that were individually incubated or mixed together and incubated anaerobically at room temperature for $30 \min$ or $2 \mathrm{~h}$. Co-migration of AprD3 and AprD4 in the native PAGE gel was not observed in this analysis. (C) Size exclusion fast protein liquid chromatography (FPLC) analysis of AprD4 and AprD3 that were individually incubated or mixed together and incubated anaerobically at room temperature for $2 \mathrm{~h}$.

A

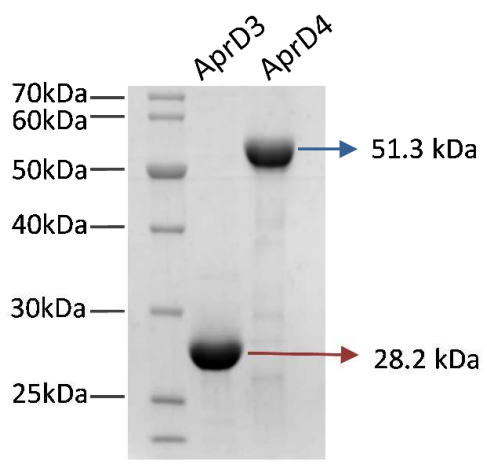

B

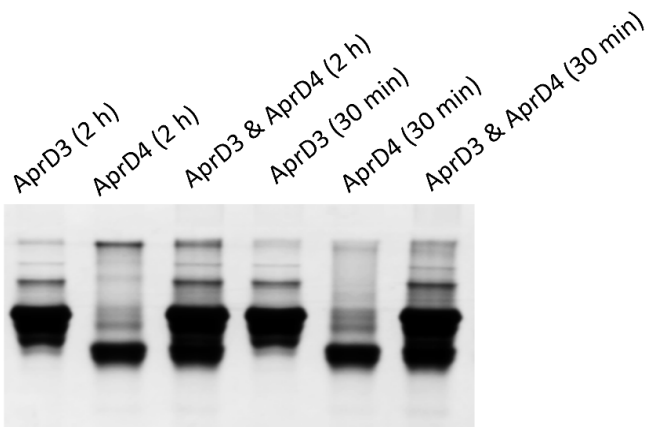

$\mathrm{C}$

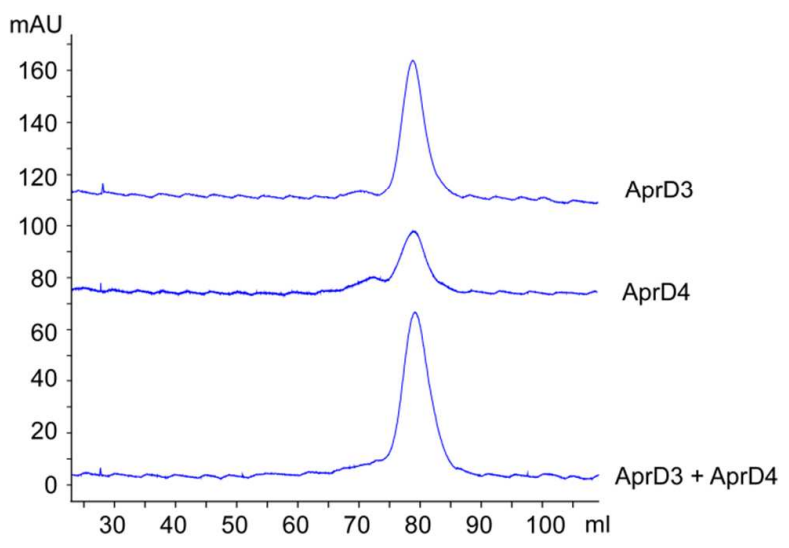


Figure S 19. Chemical structures of the representative 2-DOS-containing AGAs. The C6-amino groups or the groups derived from a C6-amino are shown in red.
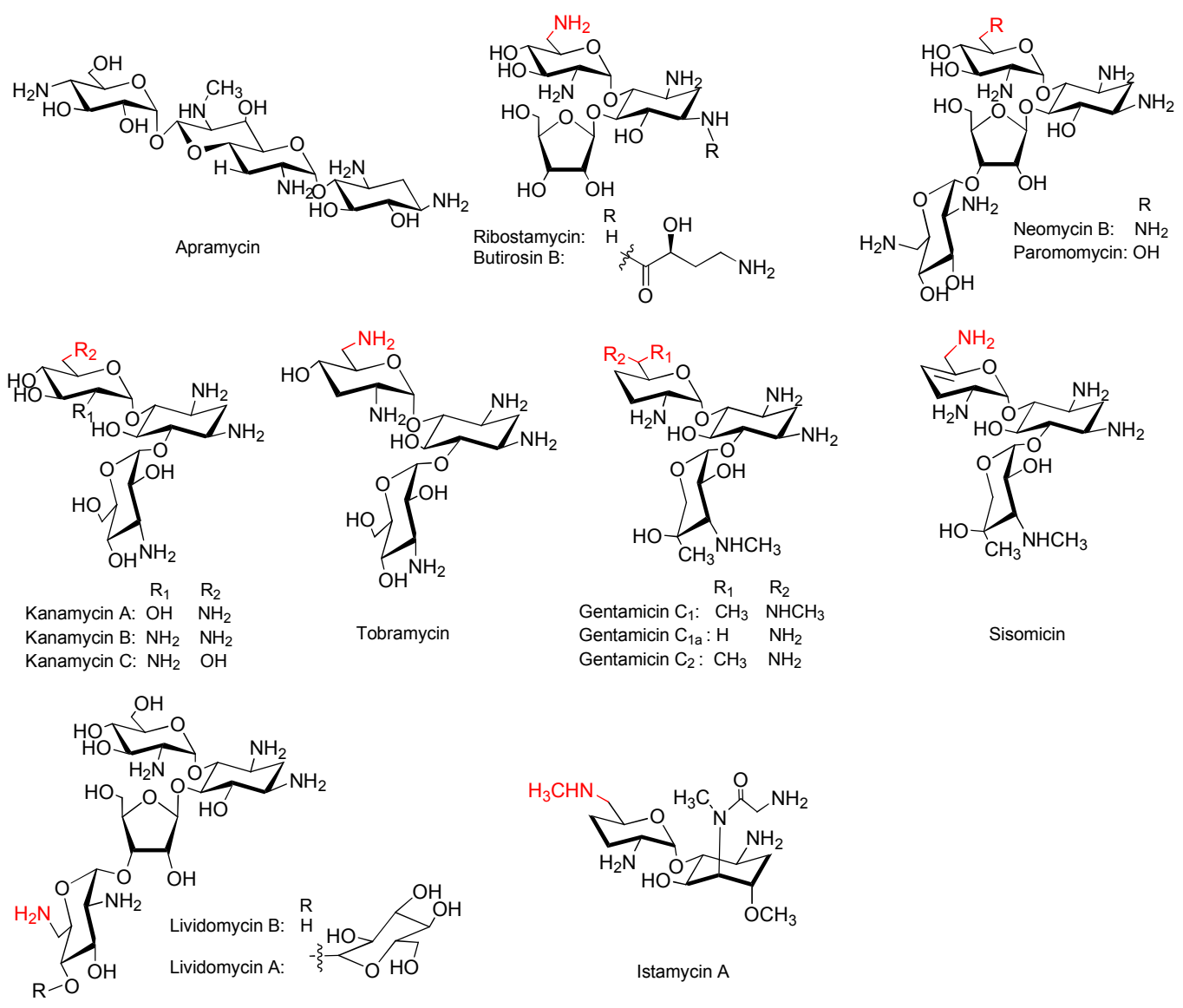

Sisomicin 
Figure S20. In-frame deletion of aprQ in S. tenebrarius, showing the schematic representation of the in-frame deletion and the southern blot analysis. The probe is shown as a purple bar, and the arrows beside the southern blot data indicate the size of the expected signal fragments.
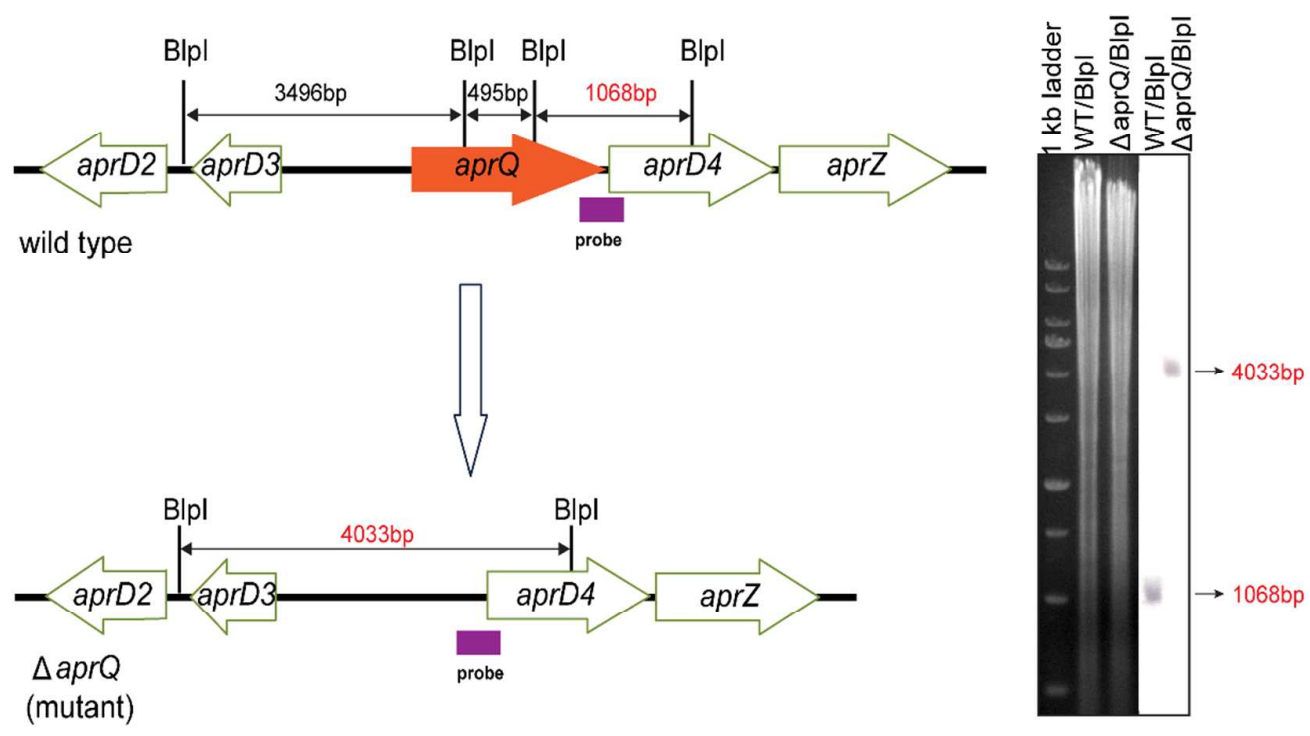
Figure S21. Generation of the $\triangle a p r Q \Delta t o b Q$ double-knockout mutant of $S$. tenebrarius, showing the schematic representation of the in-frame deletion and the southern blot analysis. The probe is shown as a purple bar, and the arrows beside the southern blot data indicate the size of the expected signal fragments.

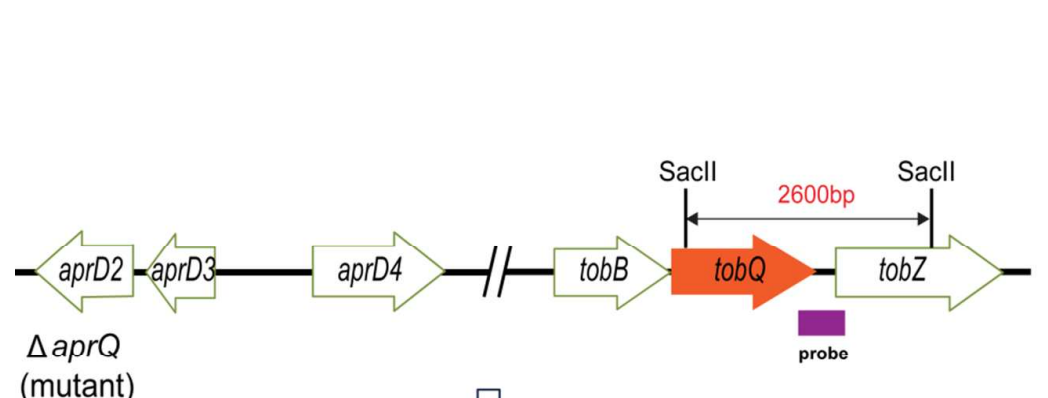
(mutant)
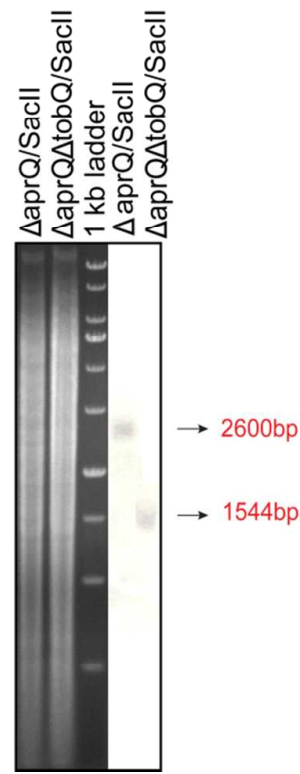
Figure S22. Generation of the $\triangle a p r D 4 \Delta t o b Q$ double-knockout mutant of $S$. tenebrarius, showing the schematic representation of the in-frame deletion and the southern blot analysis. The probe is shown as a purple bar, and the arrows beside the southern blot data indicate the size of the expected signal fragments.

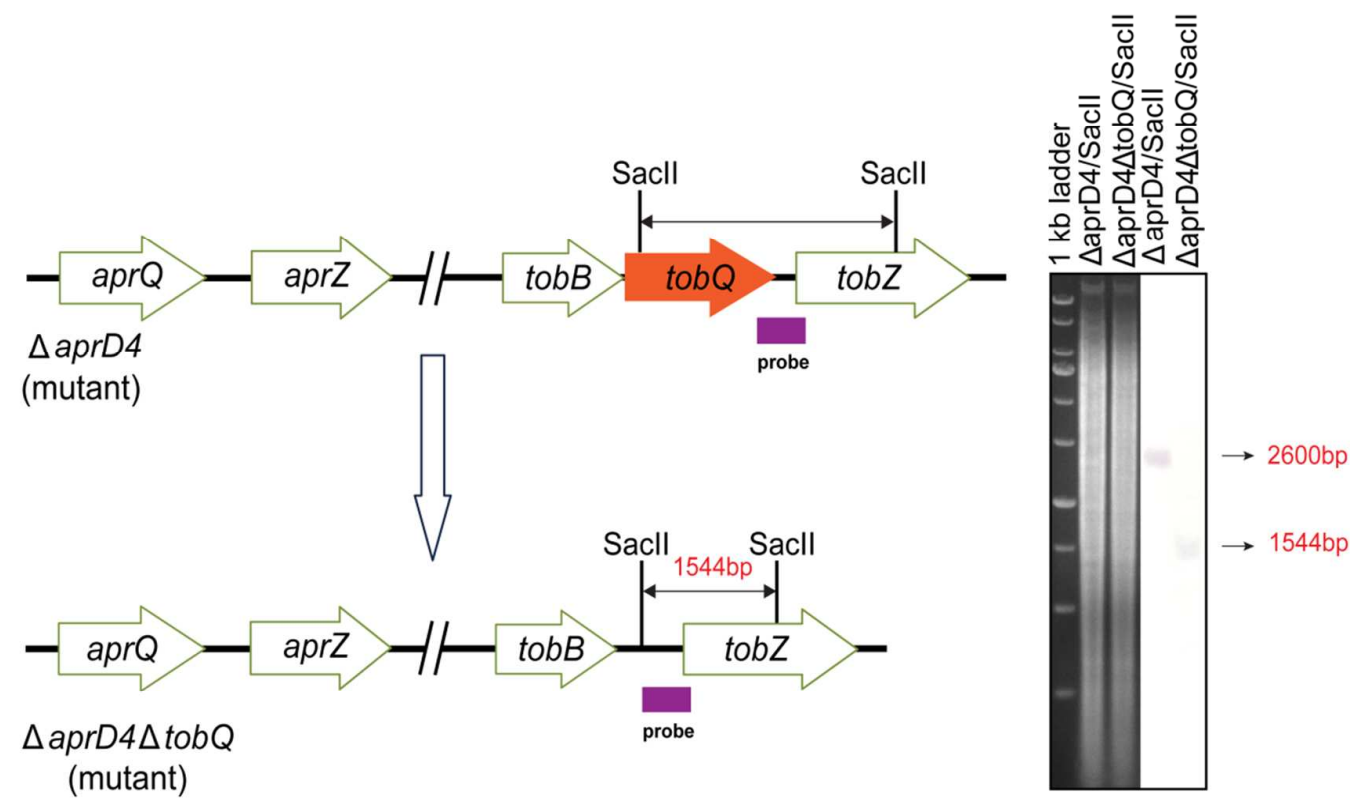


Figure S23. Generation of the $A a p r Q 4 a p r D 4$ double-knockout mutant of S. tenebrarius, showing the schematic representation of the in-frame deletion and the southern blot analysis. The probe is shown as a purple bar, and the arrows beside the southern blot data indicate the size of the expected signal fragments.
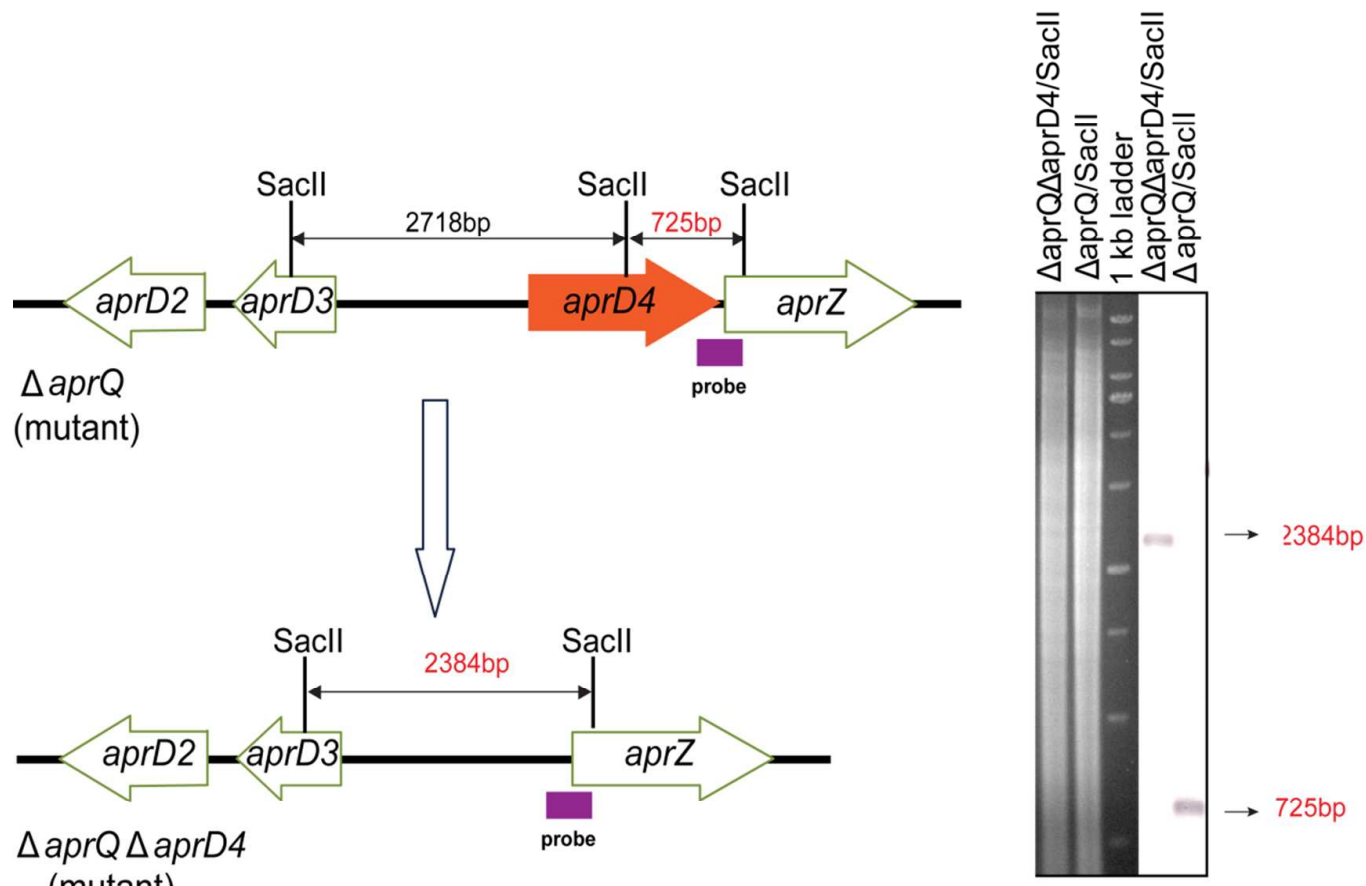

$\triangle a p r Q \Delta a p r D 4$

(mutant) 
Figure S24. SDS-PAGE analysis of the purified AprQ expressed by using Streptomyces lividans TK24 as an expression host.

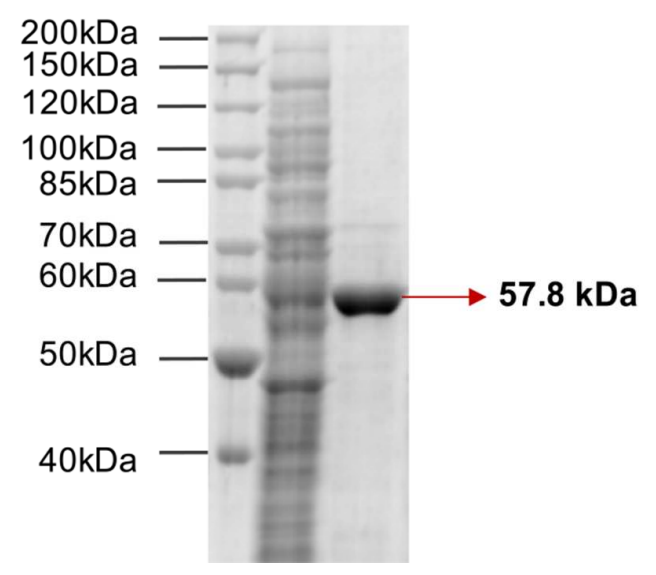


Figure S25. HR-MS/MS analysis of 6'-hydroxyl-6'-oxolividamine (HOL, 6) generated from lividamine by AprQ.

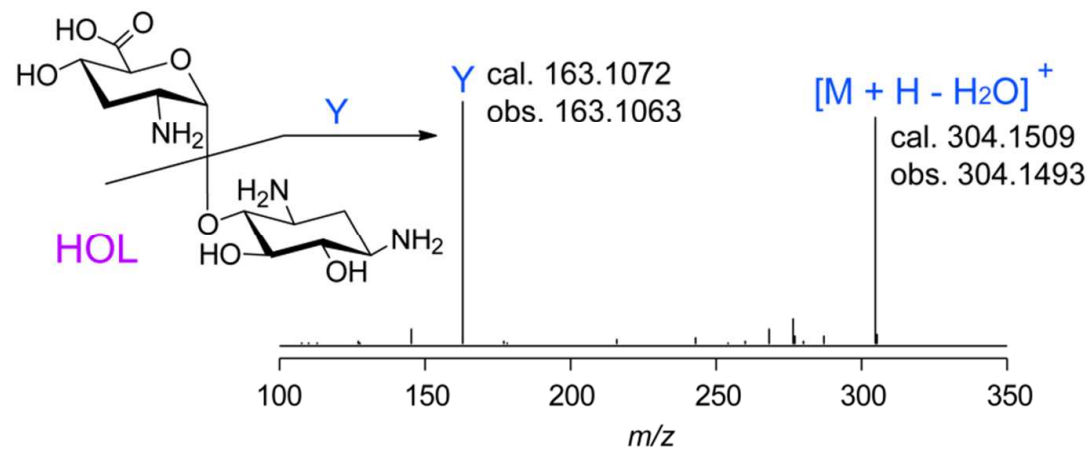


Figure S26. ${ }^{1}$ H NMR analysis of $6^{\prime}$-hydroxyl- $6^{\prime}$-oxolividamine (HOL, 6) generated from

lividamine by AprQ, showing (A) the chemical structure of HOL, and (B) the ${ }^{1} \mathrm{H}$ NMR spectrum.

A

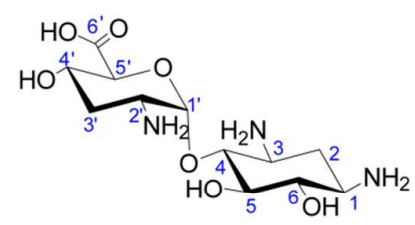

B

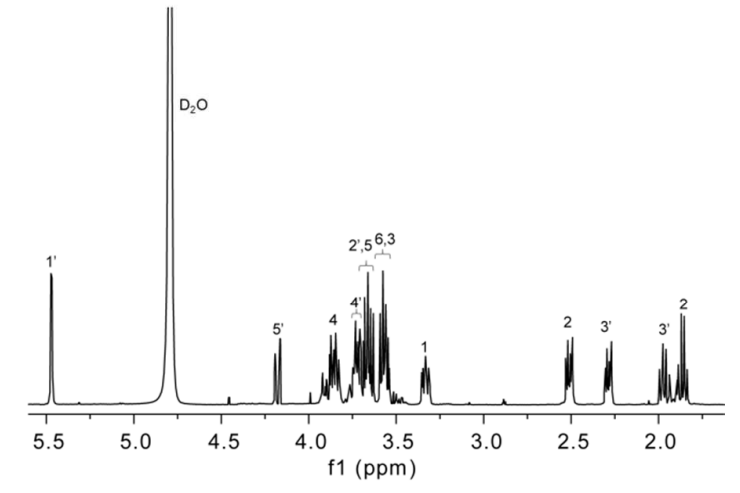




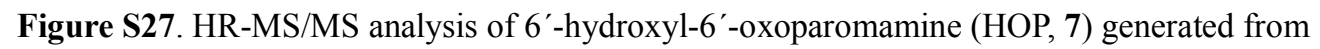
paromamine by AprQ.

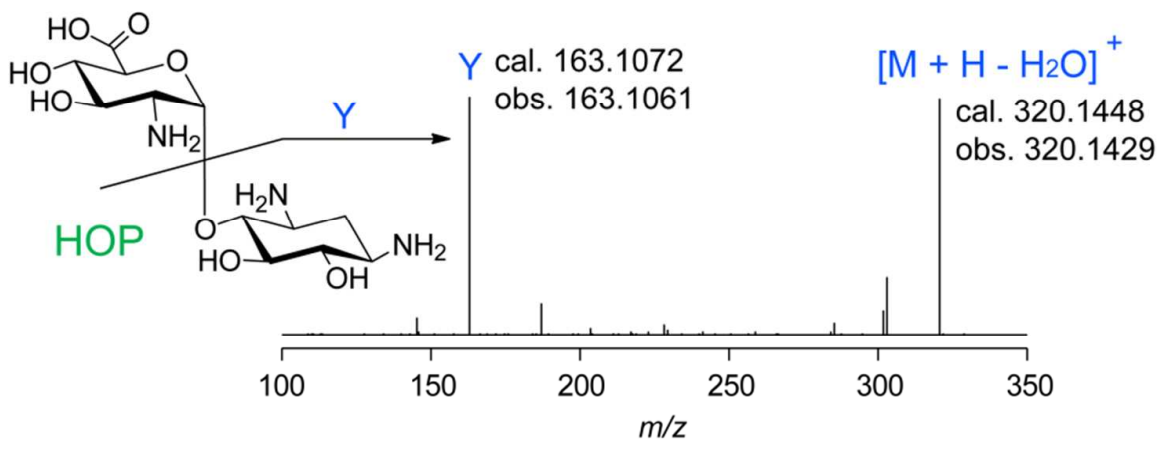


Figure S28. TobQ catalyzes the oxidation of paromamine but not lividamine. (A) LC-MS analysis of TobQ in vitro activity, showing the extracted ion chromatograms (EICs) of $[\mathrm{M}+\mathrm{H}]^{+}=322.2$ (corresponding to $6^{\prime}$-oxoparomamine, 6-OP) for (i) TobQ-catalyzed reaction with paromamine, and (ii) control reaction using paromamine and the boiled enzyme; and (iii) the EICs of $[\mathrm{M}+\mathrm{H}]^{+}$ $=306.2$ (corresponding to $6^{\prime}$-oxolividamine 6-OL) for assay with lividamine and TobQ. The assay were performed using the cell free extract of S. lividans TK24 expressing tobQ. (B) TobQ converts paromamine to 6-OP. (C) TobQ was not able to convert lividamine to 6-OL. (D) HR-MS/MS characterization of 6-OP produced in the TobQ in vitro assay.

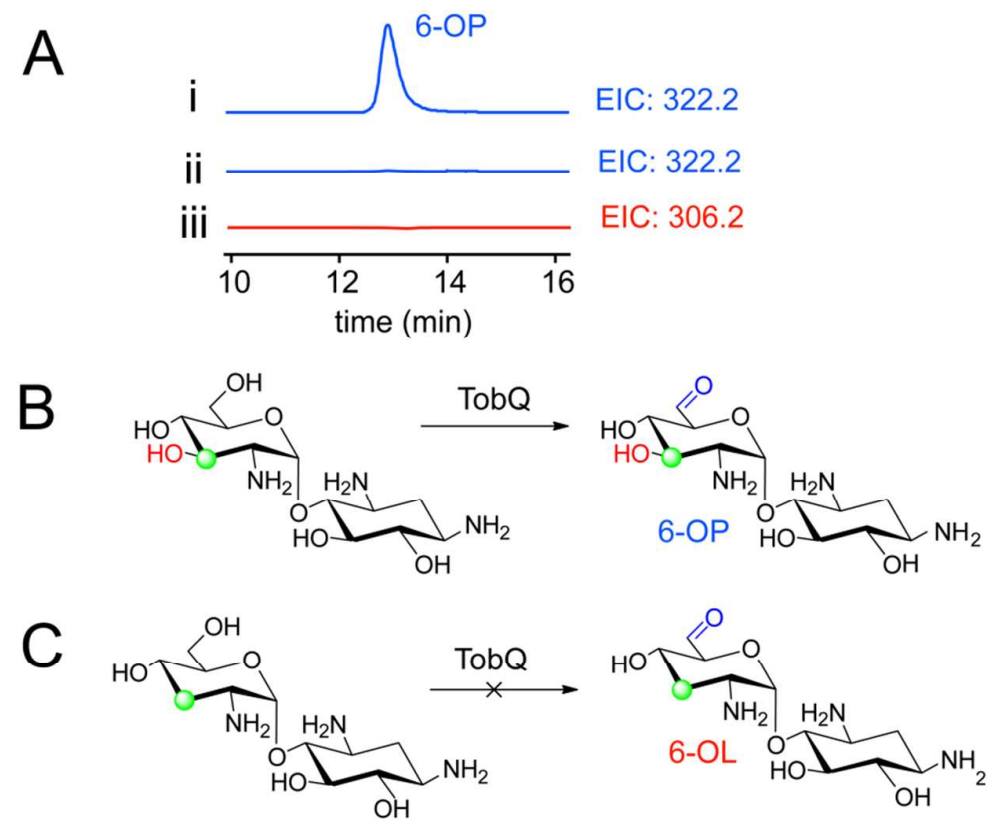

D

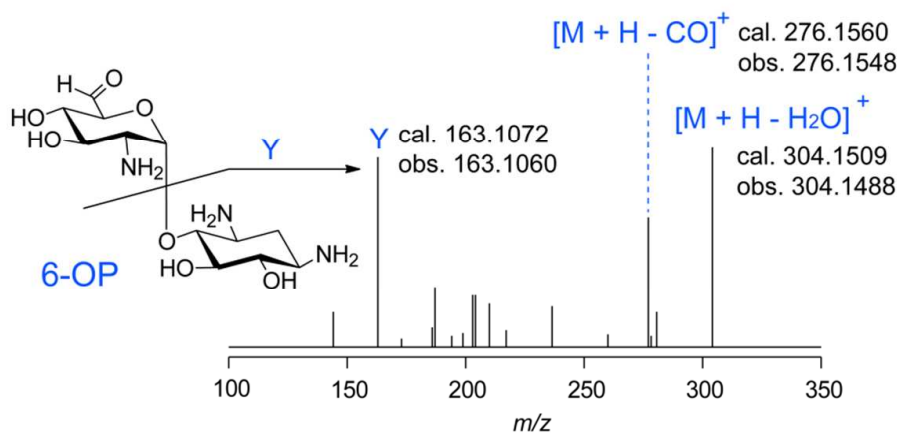


Figure S29. Unrooted Bayesian Markov chain Monte Carlo (MCMC) tree of AprQ homologous proteins. The Bayesian posterior probability (bPP) is shown along the branches, which are colored according to the taxonomies of the host organisms.

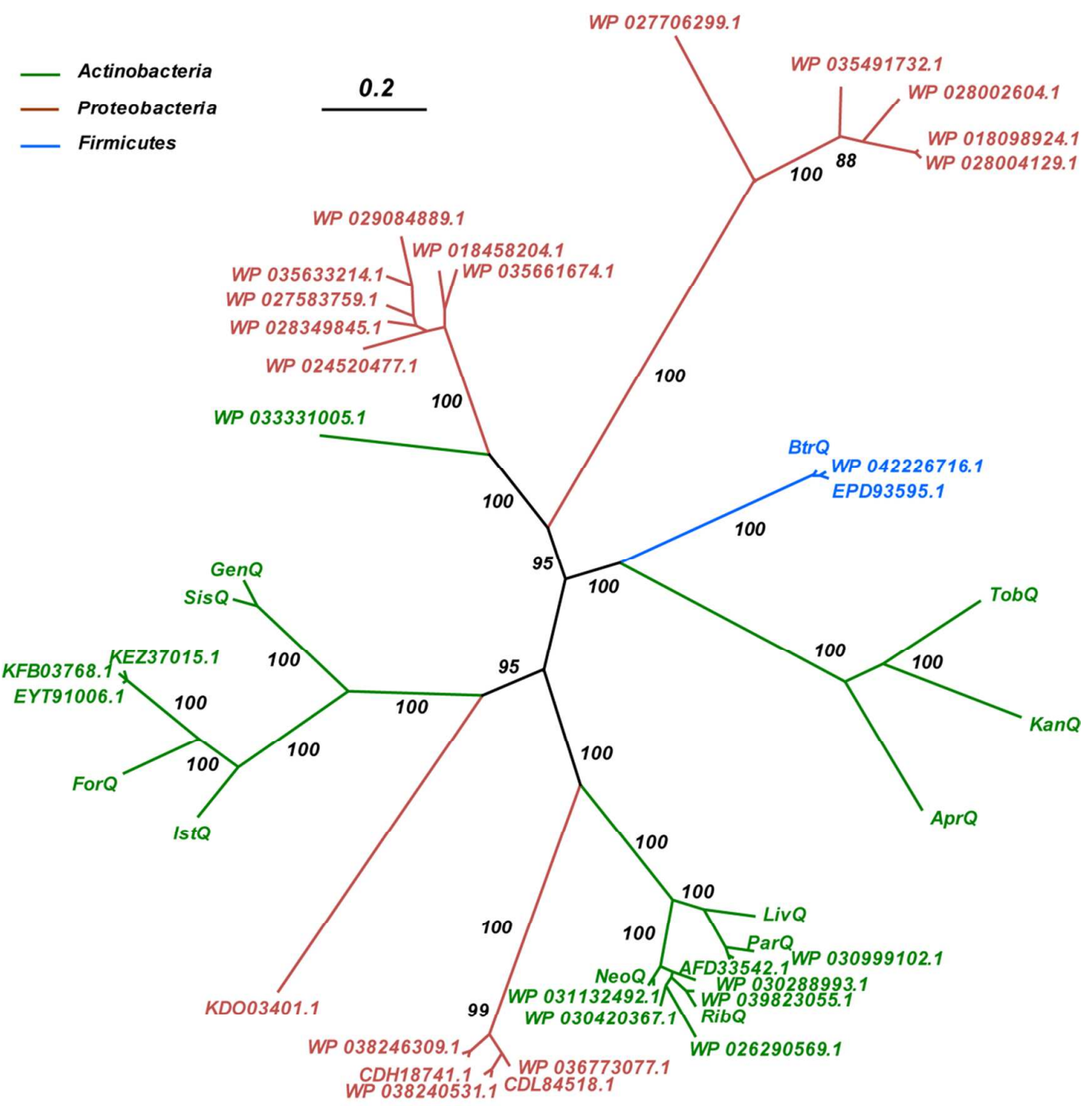


Figure S30. Summary of the GenQ in vitro activity (also see Figure S31). (A) GenQ catalyzes the oxidation of gentamicin G418 and X2. (B) GenQ is not able to catalyze the oxidation of lividamine (C) GenQ is not able to catalyze the oxidation of paromamine. 6-OL, 6'-oxolividamine; 6-OP, 6'-oxoparomamine; HOP, 6'-hydroxyl-6'-oxoparomamine; $\quad$ HOL, 6'-hydroxyl-6'-oxolividamine; 6-OGG, 6'-oxogentamicin G418; 6-OGX, 6'-oxogentamicin X2.
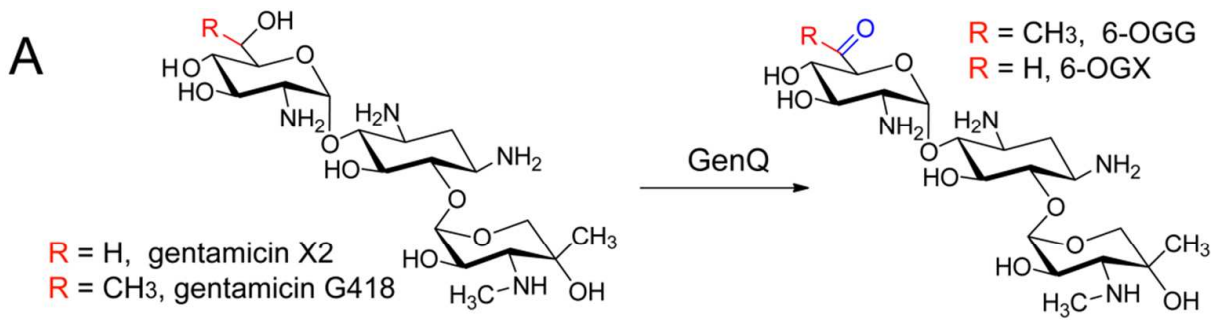

B

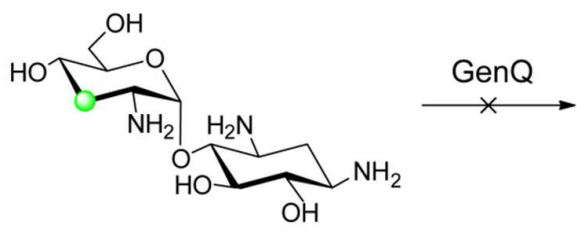

lividamine (5)
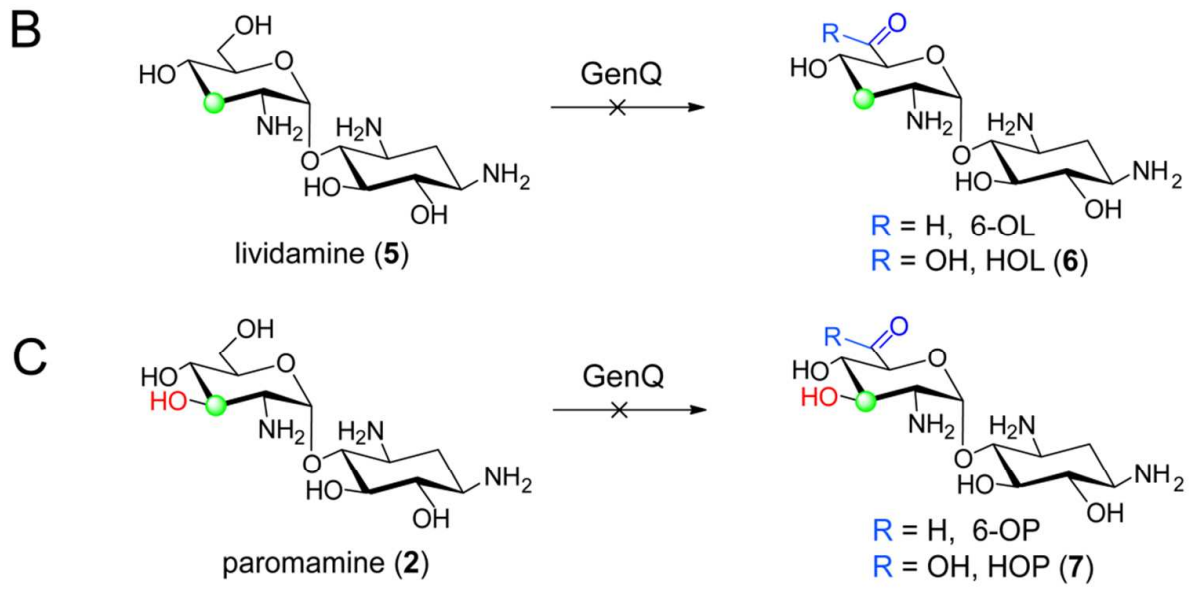
Figure S31. Biochemical study of GenQ. (A) SDS-PAGE analysis of purified GenQ. (B) LC-HR-MS analysis of GenQ in vitro assays, showing the extracted ion chromatograms (EICs) of $[\mathrm{M}+\mathrm{H}]^{+}=495.3$ (corresponding to 6-OGG) for (i) GenQ-catalyzed reaction with gentamicin G418, and (ii) control reaction using gentamicin G418 and boiled GenQ; and the EICs of [M + $\mathrm{H}]^{+}=481.3$ (corresponding to 6-OGX) for (iii) GenQ-catalyzed reaction with gentamicin X2, and (iv) control reaction using gentamicin X2 and boiled GenQ; and the EICs of (v) $[\mathrm{M}+\mathrm{H}]^{+}=322.2$ (corresponding to 6-OP) and (vi) $[\mathrm{M}+\mathrm{H}]^{+}=338.2$ (corresponding to HOP) for assay containing GenQ and paromamine; and the EICs of (vii) $[\mathrm{M}+\mathrm{H}]^{+}=306.2$ (corresponding to 6-OL) and (viii) $[\mathrm{M}+\mathrm{H}]^{+}=322.2$ (corresponding to HOL) for assay containing GenQ and lividamine. 6-OL, 6'-oxolividamine; 6-OP, 6'-oxoparomamine; HOP, 6'-hydroxyl-6'-oxoparomamine; HOL, 6'-hydroxyl-6'-oxolividamine; 6-OGG, 6'-oxogentamicin G418; 6-OGX, 6'-oxogentamicin X2.

A

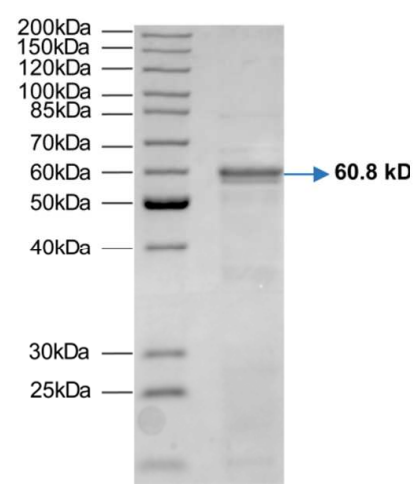

B

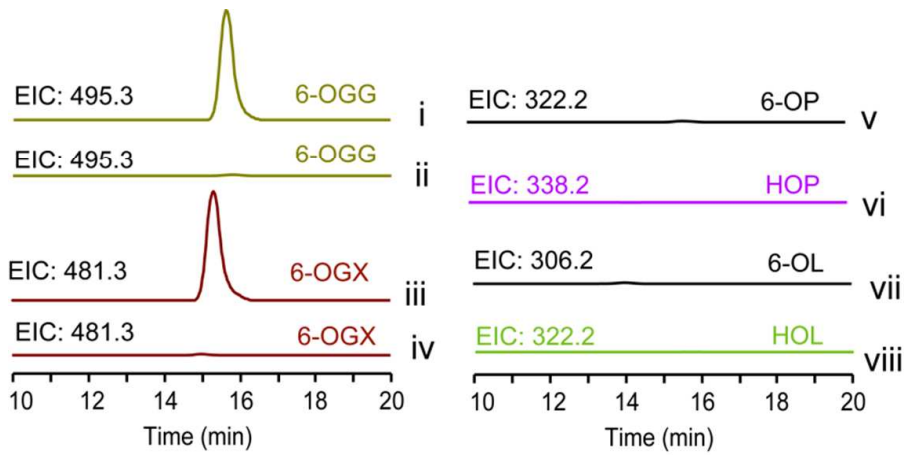


Figure S32. Comparative HR-MS/MS analysis the gentamicin analogs produced in the AprQ-catalyzed reactions, showing the spectra of 6'-hydroxyl-6'-oxogentamicin X2 (6-HOGX) and 6'-oxogentamicin G418 (6-OGG) produced in the AprQ-catalyzed reactions, and the gentamicin G418 and gentamicin X2 standards. The $\mathrm{m} / \mathrm{z}=163.10$ signals present in all 4 spectra correspond to the protonated 2-deoxystreptamine generated upon CID-induced fragmentation.
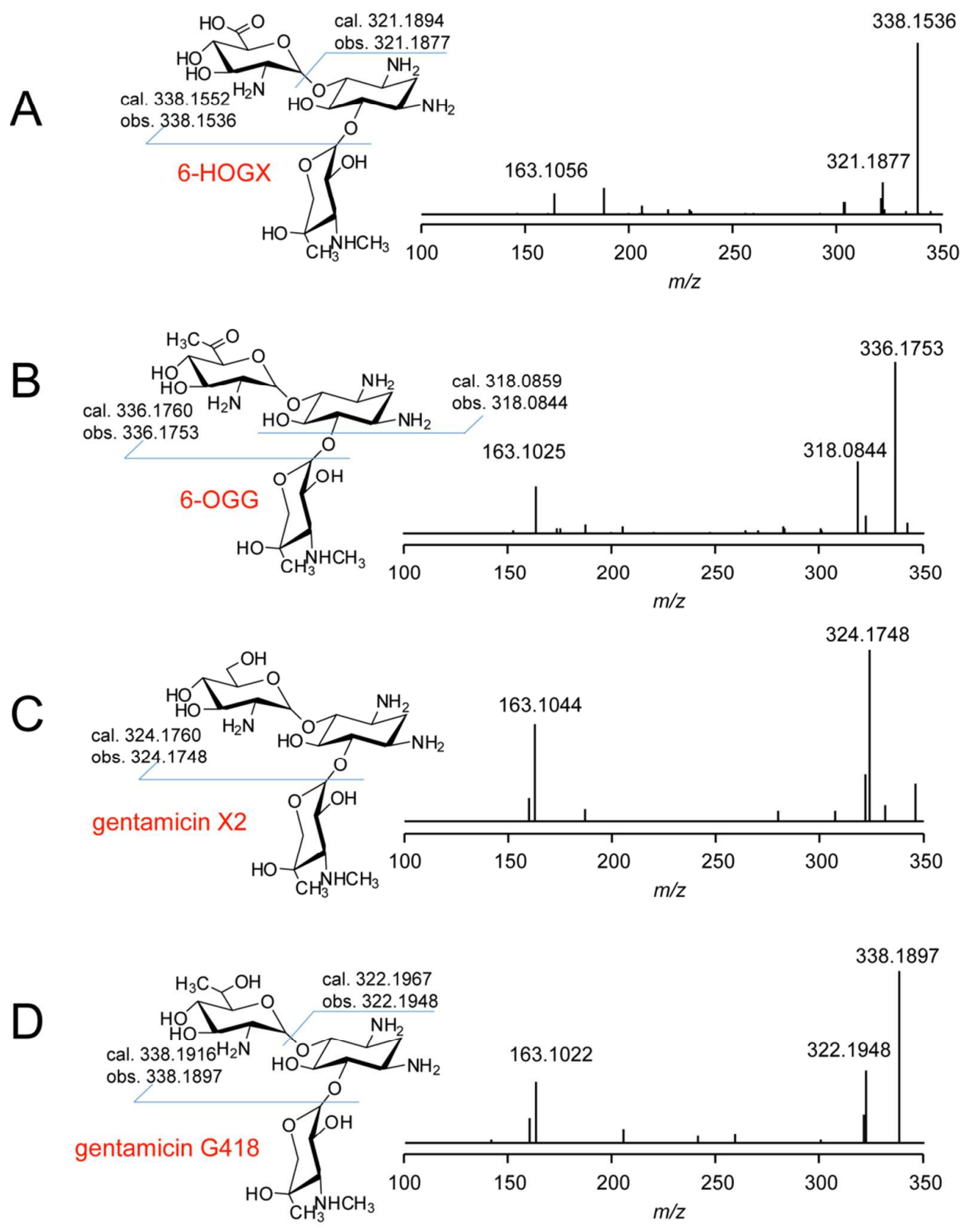
Figure S33. C3' deoxygenation of neamine by AprD4 and AprD3. (A) EICs of $[\mathrm{M}+\mathrm{H}]^{+}=307.2$ (corresponding to nebramine) for (i) reaction with AprD4 and AprD3 using neamine as the substrate, (ii) control reaction using boiled AprD4 and AprD3, and (iii) synthetic nebramine standard. (B) MS/MS spectra of (i) the compound with $[\mathrm{M}+\mathrm{H}]^{+}=307.2$ from the tandem reaction catalyzed by AprD4 and AprD3, and (ii) synthetic nebramine standard.

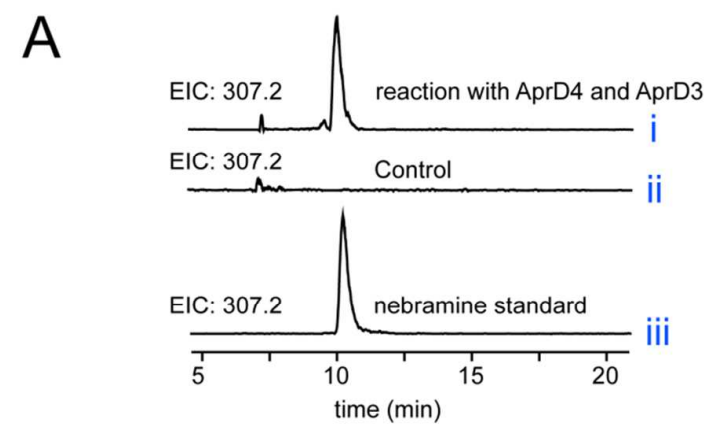

B
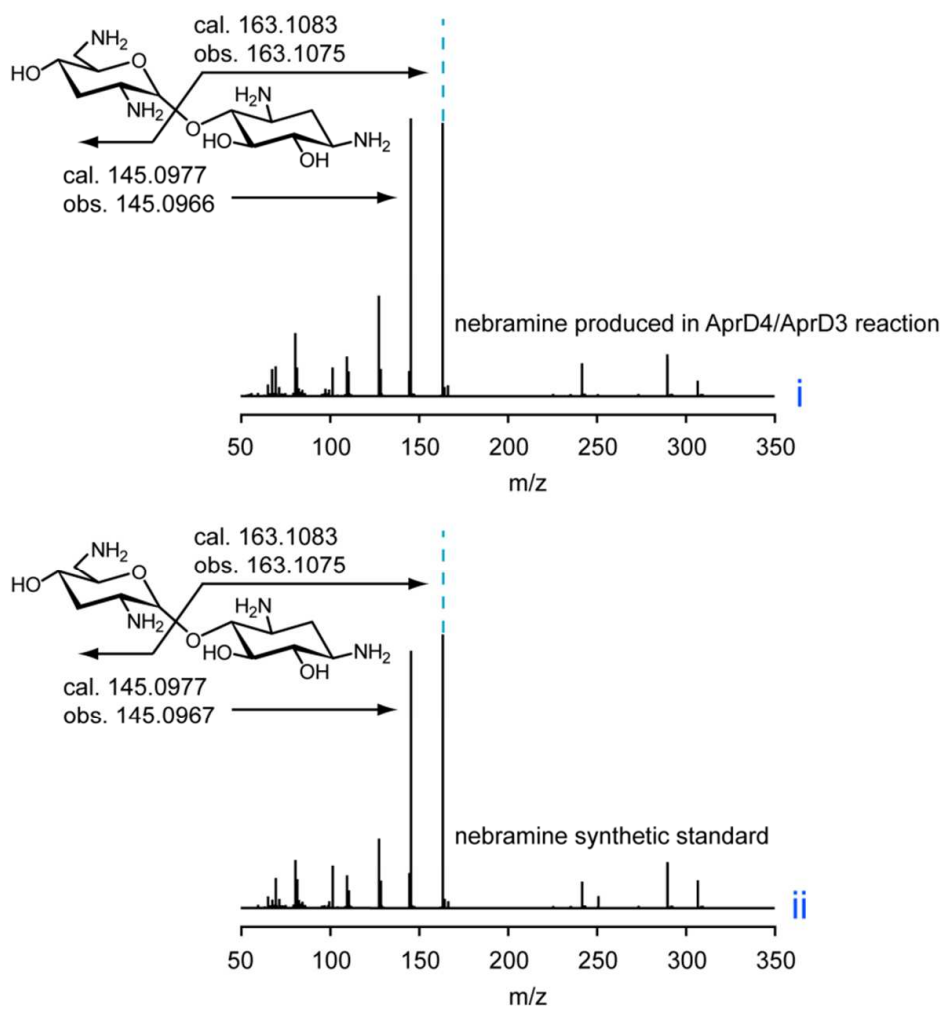
Table S1. Accession numbers of AprQ and its analogs in this study.

\begin{tabular}{|c|c|c|c|}
\hline Accession Number & Bacterial strain & Taxonomy & Name/Compound \\
\hline CAF33053.1 & Streptoalloteichus tenebrarius & Actinobacteria & AprQ/apramycin \\
\hline CAH18553.1 & Streptoalloteichus tenebrarius & Actinobacteria & TobQ/tobramycin \\
\hline CAF31587.1 & Streptomyces kanamyceticus & Actinobacteria & $\mathrm{KanQ} /$ kanamycin \\
\hline WP_042226716.1 & Paenibacillus chitinolyticus & Firmicutes & \\
\hline EPD93595.1 & Paenibacillus sp. HGH0039 & Firmicutes & \\
\hline CAG77432.1 & Bacillus circulans & Firmicutes & BtrQ/butirosin \\
\hline WP_035633214.1 & Bradyrhizobium elkanii & Proteobacteria & \\
\hline CAF32378.1 & Streptomyces rimosus & Actinobacteria & ParQ/paromomycin \\
\hline WP_030999102.1 & Streptomyces sp. NRRL WC-3773 & Actinobacteria & \\
\hline WP_024520477.1 & Bradyrhizobium sp. Tv2a-2 & Proteobacteria & \\
\hline CAF31536.1 & Micromonospora olivasterospora & Actinobacteria & ForQ/fortimicin \\
\hline WP_018458204.1 & Bradyrhizobium sp. WSM4349 & Proteobacteria & \\
\hline WP_030288993.1 & Streptomyces catenulae & Actinobacteria & \\
\hline CAG38701.1 & Streptomyces lividus & Actinobacteria & LivQ/lividomycin \\
\hline EYT91006.1 & Frankia sp. Thr & Actinobacteria & \\
\hline KFB03768.1 & Frankia sp. Allo2 & Actinobacteria & \\
\hline KEZ37015.1 & Frankia sp. $\mathrm{CeD}$ & Actinobacteria & \\
\hline WP_027583759.1 & Bradyrhizobium sp. Aila-2 & Proteobacteria & \\
\hline WP_033331005.1 & Actinomadura madurae & Actinobacteria & \\
\hline CDL84518.1 & Xenorhabdus szentirmaii DSM 16338 & Proteobacteria & \\
\hline WP_038240531.1 & Xenorhabdus szentirmaii & Proteobacteria & \\
\hline WP_038246309.1 & Xenorhabdus bovienii & Proteobacteria & \\
\hline CDH18741.1 & Xenorhabdus bovienii & Proteobacteria & \\
\hline WP_039823055.1 & Streptomyces xinghaiensis & Actinobacteria & \\
\hline WP_026290569.1 & Streptomyces $s p$. CNB091 & Actinobacteria & \\
\hline AFD33542.1 & Streptomyces xinghaiensis S187 & Actinobacteria & \\
\hline ACN38340.1 & Micromonospora inyonensis & Actinobacteria & SisQ/sisomicin \\
\hline WP_029084889.1 & Bradyrhizobium $s p$. th.b2 & Proteobacteria & \\
\hline WP_028349845.1 & Bradyrhizobium elkanii & Proteobacteria & \\
\hline WP_035661674.1 & Bradyrhizobium sp. Ec3.3 & Proteobacteria & \\
\hline WP_030420367.1 & Streptomyces $s p$. NRRL F-5065 & Actinobacteria & \\
\hline WP_036773077.1 & Photorhabdus asymbiotica & Proteobacteria & \\
\hline CAH60158.1 & Streptomyces tenjimariensis & Actinobacteria & IstQ/istamycin \\
\hline CAG34033.1 & Streptomyces ribosidificus & Actinobacteria & RibQ/ribostamycin \\
\hline WP_031132492.1 & Streptomyces sp. NRRL WC-3719 & Actinobacteria & \\
\hline CAF31426.2 & Micromonospora echinospora & Actinobacteria & GenQ/gentamicin \\
\hline CAF33316.1 & Streptomyces fradiae & Actinobacteria & NeoQ/neomycin \\
\hline KDO03401.1 & Rickettsia buchneri & Proteobacteria & \\
\hline WP_018098924.1 & Sinorhizobium meliloti & Proteobacteria & \\
\hline WP_028004129.1 & Sinorhizobium & Proteobacteria & \\
\hline WP_028002604.1 & Sinorhizobium arboris & Proteobacteria & \\
\hline WP_035491732.1 & Burkholderia sp. JPY251 & Proteobacteria & \\
\hline WP_027706299.1 & Zymobacter palmae & Proteobacteria & \\
\hline
\end{tabular}


Table S2. PCR primers used in this study.

\begin{tabular}{|c|c|c|}
\hline Primer & Sequence* & Function \\
\hline aadA-F & CTTCGGAATAGGAACTTCAT $\underline{G A G C T C}$ & $\begin{array}{c}\text { aadA } \\
\text { amplification }\end{array}$ \\
\hline aadA-R & 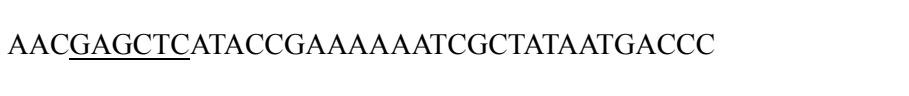 & $\begin{array}{c}\text { aadA } \\
\text { amplification }\end{array}$ \\
\hline aprD3-left-F & GGCCAGTGCCAAGCTTTCCTGGTGCTGGCCTTGGTGCTGGTGCT & $\begin{array}{l}\text { aprD3 in-frame } \\
\text { deletion }\end{array}$ \\
\hline aprD3-left-R & GTCGCGGACGTCGCGACCGACATGGGCGGTCCGGAGGCC & $\begin{array}{l}\text { aprD3 in-frame } \\
\quad \text { deletion }\end{array}$ \\
\hline aprD3-right-F & CGCGACGTCCGCGACGTCGCGGCAGGCCAGGA & $\begin{array}{l}\text { aprD3 in-frame } \\
\text { deletion }\end{array}$ \\
\hline aprD3-right-R & $\begin{array}{l}\text { ACATGATTACGAATTCAGGTAGACGCTGTTCTTCATGGCCAGGTG } \\
\text { C }\end{array}$ & $\begin{array}{l}\text { aprD3 in-frame } \\
\text { deletion }\end{array}$ \\
\hline id-aprD3-F & TGTCACCGGTTTTCCTGGGTGCTC & $\begin{array}{l}\text { WDY289 } \\
\text { verification }\end{array}$ \\
\hline id-aprD3-R & GAAGACGTGTCGGAACAGTGGAACG & $\begin{array}{l}\text { WDY289 } \\
\text { verification }\end{array}$ \\
\hline HB-aprD3-F & TCGGAATTCACGACGCCGACCACCGCGGGACGGGGGGCG & $\begin{array}{l}\triangle a p r D 3 \\
\text { complementation }\end{array}$ \\
\hline HB-aprD3-R & ACGCATATGGAGCAACGGTACGTGCTGGTCACCGGGGCC & $\begin{array}{l}\text { AaprD3 } \\
\text { complementation }\end{array}$ \\
\hline aprD4-left-F & 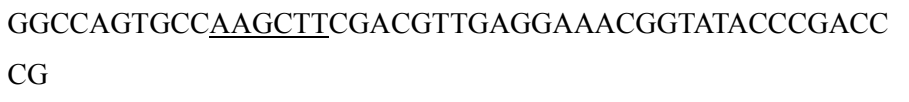 & $\begin{array}{l}\text { aprD4 in-frame } \\
\text { deletion }\end{array}$ \\
\hline aprD4aprQ-F & $\begin{array}{l}\text { GGCCAGTGCCAAGCTTGGCGTCGACCACCGCCGTCACGGCCGCG } \\
\text { TC }\end{array}$ & $\begin{array}{c}\text { aprD4aprQ } \\
\text { in-frame deletion }\end{array}$ \\
\hline aprD4-left-R & CACCGCGATCTGGTGGCAGACCGTGCCGAGCCCGCCCGC & $\begin{array}{c}\text { aprD4/aprD4apr } \\
Q \text { in-frame } \\
\text { deletion }\end{array}$ \\
\hline
\end{tabular}




\begin{tabular}{|c|c|c|}
\hline Primer & Sequence* & Function \\
\hline aprD4-right-F & CACCAGATCGCGGTGGACAAGGGGT & $\begin{array}{c}\text { aprD4/aprD4apr } \\
Q \text { in-frame } \\
\text { deletion }\end{array}$ \\
\hline aprD4-right-R & ACATGATTACGAATTCCAGGCCGTGGTTGCGCAGCAGCCG & $\begin{array}{c}\text { aprD4/aprD4apr } \\
Q \text { in-frame } \\
\text { deletion }\end{array}$ \\
\hline id-aprD4-F & TCTTCCCCTTCGCCGGCAACCTCAA & $\begin{array}{l}\text { WDY288 } \\
\text { verification }\end{array}$ \\
\hline id-aprD4-R & GGTCGCCGATGCTCAGCTCGTCGGT & $\begin{array}{l}\text { WDY288 } \\
\text { verification }\end{array}$ \\
\hline HB-AprD4-F & AATCATATGCGACGAATGCGGCTCGGCACGGTACTGCTG & $\begin{array}{l}\Delta a p r D 4 \\
\text { complementation }\end{array}$ \\
\hline HB-AprD4-R & CCAGAATTCAGGCGTCACCGGTCGACCAGGCGTGCACCC & $\begin{array}{l}\Delta \text { aprD4 } \\
\text { complementation }\end{array}$ \\
\hline aprQ-left-F & GGCCAGTGCCAAGCTTCGGGAGGGTTCGTGGGGAATGGGG & $\begin{array}{l}\text { aprQ in-frame } \\
\text { deletion }\end{array}$ \\
\hline aprQ-left-R & CCGCTCGGCCAGAGTGAACGCCGTGA & $\begin{array}{l}\operatorname{apr} Q \text { in-frame } \\
\text { deletion }\end{array}$ \\
\hline aprQ-right-F & GACTACGACAACGTGTGGGCGGTCG & $\begin{array}{l}\text { aprQ in-frame } \\
\text { deletion }\end{array}$ \\
\hline aprQ-right-R & ACATGATTACGAATTCCGCCGCCCATCATCACGTCGGCCC & $\begin{array}{l}\text { aprQ in-frame } \\
\text { deletion }\end{array}$ \\
\hline id-aprQ-id-F & ACCTCCGTTCGAGTGACAATGGTC & $\begin{array}{l}\text { WDY290 } \\
\text { verification }\end{array}$ \\
\hline id-aprQ-R & GCCAGGCGCATCAGGTCGAGCGGG & $\begin{array}{l}\text { WDY290 } \\
\text { verification }\end{array}$ \\
\hline HB-aprQ-F & GTAGGATCCACATATGAGGTTCGTGACGCTCCAGGAGGCCACC & $\begin{array}{c}\triangle a p r Q \\
\text { complementation }\end{array}$ \\
\hline HB-aprQ-R & GACATGATTACGAATTCGGAACCGGGGGTGTCAGGAGATGGCG & $\begin{array}{c}\triangle \operatorname{AaprQ} \\
\text { complementation }\end{array}$ \\
\hline tobQ-left-F & $\begin{array}{l}\text { GGCCAGTGCCAAGCTTGACTCTGTCGCCGGCCGTGATGTGTCCA } \\
\text { C }\end{array}$ & $\begin{array}{l}\text { tobQ in-frame } \\
\text { deletion }\end{array}$ \\
\hline tobQ-left-R & CAAGGCAGGGGCGATCGACGTGCGGGAGGTGGACA & $\begin{array}{l}\text { tobQ in-frame } \\
\text { deletion }\end{array}$ \\
\hline tobQ-right-F & $\begin{array}{l}\text { ATCGCCCCTGCCTTGCCGTCGCGCGTCCACCGCCCGTGGCGACG } \\
\text { C }\end{array}$ & $\begin{array}{l}\text { tob } Q \text { in-frame } \\
\text { deletion }\end{array}$ \\
\hline
\end{tabular}




\begin{tabular}{|c|c|c|}
\hline Primer & Sequence* & Function \\
\hline tobQ-right-R & ACATGATTACGAATTCGCACAGCATGACGGCGATCGACGAGTAC & $\begin{array}{l}\text { tobQ in-frame } \\
\text { deletion }\end{array}$ \\
\hline id-tobQ-F & CCGCCTGCACCGGGGCCGTGTTGTAC & $\begin{array}{l}\text { WDY291 and } \\
\text { WDY292 } \\
\text { verification }\end{array}$ \\
\hline id-tobQ-R & CCCTGCTGCGGCACGGCGTCTTCTG & $\begin{array}{l}\text { Same to } \\
\text { id-tobQ-F }\end{array}$ \\
\hline HB-tobQ-F & GTAGGATCCACATATGCTGCTGATCTCAGCGGACGAGGCCGCGC & $\begin{array}{l}\Delta t o b Q \\
\text { complementation }\end{array}$ \\
\hline HB-tobQ-R & GACATGATTACGAATTCGGAACCGGTGTGAGGAACCGGTGTGGC & $\begin{array}{l}\Delta t o b Q \\
\text { complementation }\end{array}$ \\
\hline pGM1190_F & $\begin{array}{l}\text { AGAGAAGGGAGCGGACCATGGGCAGCAGCCATCATCATCATCAT } \\
\text { C }\end{array}$ & $\begin{array}{l}\text { pWDYHS01 } \\
\text { construction }\end{array}$ \\
\hline pGM1190_R & AAACTTTAGATCTGGGTTAGCAGCCGGATCTCAGTGGTGG & $\begin{array}{l}\text { pWDYHS01 } \\
\text { construction }\end{array}$ \\
\hline N-AprD3-F & $\begin{array}{l}\text { AAGGAGATATACCATGGGCCATCATCATCATCATCACATGGAGCA } \\
\text { ACGGTACGTGCTGG }\end{array}$ & $\begin{array}{c}\text { AprD3 } \\
\text { overexpression } \\
\text { (His-Tagged) }\end{array}$ \\
\hline $\mathrm{N}-A p r D 3-\mathrm{R}$ & $\begin{array}{l}\text { GGTGGTGGTGCTCGAGTTATTATCACGACGCCGACCACCGCGGG } \\
\text { AC }\end{array}$ & $\begin{array}{c}\text { AprD3 } \\
\text { overexpression } \\
\text { (His-Tagged) }\end{array}$ \\
\hline N-AprD4-F & $\begin{array}{l}\text { AAGGAGATATACCATGGGCCATCATCATCATCATCACGTGAATGA } \\
\text { GGTGCGACGAATGC }\end{array}$ & $\begin{array}{c}\text { AprD4 } \\
\text { overexpression }\end{array}$ \\
\hline N-AprD4-R & $\begin{array}{l}\text { GGTGGTGGTGCTCGAGTTATTATCAGGCGTCACCGGTCGACCAG } \\
\text { GC }\end{array}$ & $\begin{array}{c}\text { AprD4 } \\
\text { overexpression }\end{array}$ \\
\hline $\begin{array}{l}\text { N-flag-AprD3- } \\
\text { F }\end{array}$ & $\begin{array}{l}\text { ATACCATGGGCGACTACAAGGACGACGATGACAAAATGGAGCAA } \\
\text { CGGTACGTGCTGGTC }\end{array}$ & $\begin{array}{c}\text { AprD3 } \\
\text { overexpression } \\
\text { (Flag-Tagged) }\end{array}$ \\
\hline N-AprQ-F & $\begin{array}{l}\text { GCGCGGCAGCCATATGATGAGGTTCGTGACGCTCCAGGAGGCCA } \\
\text { CC }\end{array}$ & $\begin{array}{l}\text { AprQ } \\
\text { overexpression }\end{array}$ \\
\hline N-AprQ-R & $\begin{array}{l}\text { GGTGGTGGTGCTCGAGTTATTATCAGGAGATGGCGCAGGCGATCC } \\
\text { G }\end{array}$ & $\begin{array}{c}\text { AprQ } \\
\text { overexpression }\end{array}$ \\
\hline N-TobQ-F & AGGCATATGCTGCTGATCTCAGCGGACGAGGCCGC & $\begin{array}{c}\text { TobQ } \\
\text { overexpression }\end{array}$ \\
\hline N-TobQ-R & $\begin{array}{l}\text { GGTGGTGGTGCTCGAGTTATTATCAGTCGATCGCGGACGCGATCC } \\
\text { G }\end{array}$ & $\begin{array}{c}\text { TobQ } \\
\text { overexpression }\end{array}$ \\
\hline $\begin{array}{l}\text { AprD4 } \\
\text { C203A-F }\end{array}$ & CGGCAGCTTCGGCGCCCCCTACCCGTGCC & $\begin{array}{c}\text { AprD4 } \\
\text { mutagenesis }\end{array}$ \\
\hline AprD4 & GGCACGGGTAGGGGGCGCCGAAGCTGCCG & AprD4 \\
\hline
\end{tabular}




\begin{tabular}{|c|c|c|}
\hline Primer & Sequence* & Function \\
\hline C203A-R & & mutagenesis \\
\hline $\begin{array}{c}\text { AprD4 } \\
\text { C207A-F }\end{array}$ & CTGCCCCTACCCGGCCCGCTTCTACTGCC & $\begin{array}{c}\text { AprD4 } \\
\text { mutagenesis }\end{array}$ \\
\hline $\begin{array}{c}\text { AprD4 } \\
\text { C207A-R }\end{array}$ & GGCAGTAGAAGCGGGCCGGGTAGGGGCAG & $\begin{array}{c}\text { AprD4 } \\
\text { mutagenesis }\end{array}$ \\
\hline $\begin{array}{c}\text { AprD4 } \\
\text { C211A-F }\end{array}$ & CGTGCCGCTTCTACGCCCCGTACCCGCTC & $\begin{array}{c}\text { AprD4 } \\
\text { mutagenesis }\end{array}$ \\
\hline $\begin{array}{c}\text { AprD4 } \\
\text { C211A-R }\end{array}$ & GAGCGGGTACGGGGCGTAGAAGCGGCACG & $\begin{array}{c}\text { AprD4 } \\
\text { mutagenesis }\end{array}$ \\
\hline AprD4 C31A-F & GGGCTCGGCACGGTCGCCAAGGACGAGGACTTC & $\begin{array}{c}\text { AprD4 } \\
\text { mutagenesis }\end{array}$ \\
\hline AprD4 C31A-R & GAAGTCCTCGTCCTTGGCGACCGTGCCGAGCCC & $\begin{array}{c}\text { AprD4 } \\
\text { mutagenesis }\end{array}$ \\
\hline AprD4 C79A-F & GGCACCACGGTGATCGCCCACGTCTCGCTGCC & $\begin{array}{c}\text { AprD4 } \\
\text { mutagenesis }\end{array}$ \\
\hline AprD4 C79A-R & GGCAGCGAGACGTGGGCGATCACCGTGGTGCC & $\begin{array}{c}\text { AprD4 } \\
\text { mutagenesis }\end{array}$ \\
\hline $\begin{array}{c}\text { AprD4 } \\
\text { C103A-F }\end{array}$ & CGCAGGGCGCCCGCGCCTACGCCTACACG & $\begin{array}{c}\text { AprD4 } \\
\text { mutagenesis }\end{array}$ \\
\hline $\begin{array}{c}\text { AprD4 } \\
\text { C103A-R }\end{array}$ & CGTGTAGGCGTAGGCGCGGGCGCCCTGCG & $\begin{array}{c}\text { AprD4 } \\
\text { mutagenesis }\end{array}$ \\
\hline $\begin{array}{c}\text { AprD4 } \\
\text { C124A-F }\end{array}$ & GAGCGGGGCGGCGCCGAGGGCGTCCTG & $\begin{array}{c}\text { AprD4 } \\
\text { mutagenesis }\end{array}$ \\
\hline $\begin{array}{c}\text { AprD4 } \\
\text { C124A-R }\end{array}$ & CAGGACGCCCTCGGCGCCGCCCCGCTC & $\begin{array}{c}\text { AprD4 } \\
\text { mutagenesis }\end{array}$ \\
\hline $\begin{array}{c}\text { AprD4 } \\
\text { C236A-F }\end{array}$ & CGGAGTTCCGGCAGGCCGCCGAGCTGGGC & $\begin{array}{c}\text { AprD4 } \\
\text { mutagenesis }\end{array}$ \\
\hline $\begin{array}{c}\text { AprD4 } \\
\text { C236A-R }\end{array}$ & GCCCAGCTCGGCGGCCTGCCGGAACTCCG & $\begin{array}{c}\text { AprD4 } \\
\text { mutagenesis }\end{array}$ \\
\hline $\begin{array}{c}\text { AprD4 } \\
\text { C262A-F }\end{array}$ & CGCACCCTGGAGCTGGCCCAGGCCCTCAAGG & $\begin{array}{c}\text { AprD4 } \\
\text { mutagenesis }\end{array}$ \\
\hline $\begin{array}{c}\text { AprD4 } \\
\text { C262A-R }\end{array}$ & CCTTGAGGGCCTGGGCCAGCTCCAGGGTGCG & $\begin{array}{c}\text { AprD4 } \\
\text { mutagenesis }\end{array}$ \\
\hline $\begin{array}{c}\text { AprD4 } \\
\text { C276A-F }\end{array}$ & GGGTGCCCTGGTGGGCCGAGACCCGGATCG & $\begin{array}{c}\text { AprD4 } \\
\text { mutagenesis }\end{array}$ \\
\hline $\begin{array}{c}\text { AprD4 } \\
\text { C276A-R }\end{array}$ & CGATCCGGGTCTCGGCCCACCAGGGCACCC & $\begin{array}{c}\text { AprD4 } \\
\text { mutagenesis }\end{array}$ \\
\hline $\begin{array}{c}\text { AprD4 } \\
\text { C296A-F }\end{array}$ & GGTCGACGCCGGGGCCGTCGGCGTCGAG & $\begin{array}{c}\text { AprD4 } \\
\text { mutagenesis }\end{array}$ \\
\hline $\begin{array}{c}\text { AprD4 } \\
\text { C296A-R }\end{array}$ & CTCGACGCCGACGGCCCCGGCGTCGACC & $\begin{array}{c}\text { AprD4 } \\
\text { mutagenesis }\end{array}$ \\
\hline
\end{tabular}




\begin{tabular}{|c|l|c|}
\hline Primer & Sequence* & Function \\
\hline AprD4 & GCTGCACGCGGTCGCCAAGGCGGGGCCC & AprD4 \\
C432A-F & & mutagenesis \\
\hline AprD4 & GGGCCCCGCCTTGGCGACCGCGTGCAGC & AprD4 \\
C432A-R & & mutagenesis \\
\hline
\end{tabular}

*Restriction sites are underlined. 
Table S3. Plasmids (for Streptomyces genetics) and Streptomyces strains used in this study.

\begin{tabular}{|c|c|}
\hline Strain/plasmid & Relevant genotype/comments \\
\hline \multicolumn{2}{|l|}{ Plasmids } \\
\hline pWHU258 & $\operatorname{aac}(3) I V$ was replaced by aadA in pOJ 260 \\
\hline pWHU268 & $a a c(3) I V$ was replaced by aadA in pIB139 \\
\hline pWDYHS01 & $\begin{array}{l}\text { His }_{6} \text {-tag was inserted downstream of tipA promoter in } \\
\text { protein overexpression vector in Streptomyces (tsr, } \\
\text { aphII, PtipA promoter, RBS, N/C-terminal } \text { His }_{6} \text {-tag, fd } \\
\text { terminator) }\end{array}$ \\
\hline pWHU288 & aprD4 in-frame deletion construction \\
\hline pWHU289 & aprD3 in-frame deletion construction \\
\hline pWHU290 & aprQ in-frame deletion construction \\
\hline pWHU291 & tobQ in-frame deletion construction \\
\hline pWHU293 & aprD4aprQ double in-frame deletion construction \\
\hline pWHU300 & aprD4 complementation construction \\
\hline pWHU301 & aprD3 complementation construction \\
\hline pWHU302 & aprQ complementation construction \\
\hline pWHU303 & tobQ complementation construction \\
\hline pWHU320 & AprQ overexpression construction \\
\hline pWHU321 & TobQ overexpression construction \\
\hline \multicolumn{2}{|l|}{ Streptomyces } \\
\hline S. tenebrarius & apramycin producer strain \\
\hline WDY288 & aprD4 in-frame deletion mutant \\
\hline WDY289 & aprD3 in-frame deletion mutant \\
\hline WDY290 & aprQ in-frame deletion mutant \\
\hline WDY291 & tobQ in-frame deletion mutant \\
\hline WDY292 & aprQ tobQ double in-frame deletion mutant \\
\hline WDY293 & aprD4 aprQ double in-frame deletion mutant \\
\hline WDY294 & aprD4 tobQ double in-frame deletion mutant \\
\hline WDY300 & aprD4 complementation mutant \\
\hline WDY301 & aprD3 complementation mutant \\
\hline WDY302 & aprQ complementation mutant \\
\hline WDY303 & tobQ complementation mutant \\
\hline WDY306 & aprQ complemented to WDY292 mutant \\
\hline WDY307 & aprQ complemented to WDY293 mutant \\
\hline WDY308 & tobQ complemented to WDY293 mutant \\
\hline WDY320 & AprQ overexpression in S. lividans TK24 \\
\hline WDY321 & TobQ overexpression in S. lividans TK24 \\
\hline
\end{tabular}




\section{References}

(1) Guo, J.; Huang, F.; Huang, C.; Duan, X.; Jian, X.; Leeper, F.; Deng, Z.; Leadlay, P. F.; Sun, Y. Chem Biol 2014, 21, 608.

(2) Li, R.; Zhu, H.; Ruan, J.; Qian, W.; Fang, X.; Shi, Z.; Li, Y.; Li, S.; Shan, G.; Kristiansen, K.; Yang, H.; Wang, J. Genome Res. 2010, 20, 265.

(3) Gust, B.; Challis, G. L.; Fowler, K.; Kieser, T.; Chater, K. F. Proc Natl Acad Sci U S A 2003, 100, 1541.

(4) Bierman, M.; Logan, R.; O'Brien, K.; Seno, E. T.; Rao, R. N.; Schoner, B. E. Gene 1992, 116, 43.

(5) Wilkinson, C. J.; Hughes-Thomas, Z. A.; Martin, C. J.; Bohm, I.; Mironenko, T.; Deacon, M.; Wheatcroft, M.; Wirtz, G.; Staunton, J.; Leadlay, P. F. J Mol Microbiol Biotechnol 2002, 4, 417.

(6) Kieser, T.; Bibb, M. J.; Buttner, M. J.; Chater, K. F.; Hopwood, D. A. Practical Streptomyces genetics 2000, The John Innes Foundation.

(7) Thorpe, H. M.; Smith, M. C. Proc Natl Acad Sci U S A 1998, 95, 5505.

(8) Combes, P.; Till, R.; Bee, S.; Smith, M. C. J Bacteriol 2002, 184, 5746.

(9) Zheng, L.; Baumann, U.; Reymond, J. L. Nucleic Acids Research 2004, 32.

(10) Muth, G.; Nussbaumer, B.; Wohlleben, W.; Puhler, A. Mol Gen Genet 1989, 219, 341.

(11) Thompson, J. D.; Gibson, T. J.; Plewniak, F.; Jeanmougin, F.; Higgins, D. G. Nucleic Acids Res $1997,25,4876$.

(12) Ronquist, F.; Teslenko, M.; van der Mark, P.; Ayres, D. L.; Darling, A.; Hohna, S.; Larget, B.; Liu, L.; Suchard, M. A.; Huelsenbeck, J. P. Syst Biol 2012, 61, 539.

(13) Page, R. D. Comput Appl Biosci 1996, 12, 357.

(14) Ko, Y.; Ruszczycky, M. W.; Choi, S. H.; Liu, H. W. Angew Chem Int Ed Eng/ 2015, 54, 860.

(15) Lin, G. M.; Choi, S. H.; Ruszczycky, M. W.; Liu, H. W. J Am Chem Soc 2015, 137, 4964.

(16) Ji, X. J.; Li, Y. Z.; Jia, Y. L.; Ding, W.; Zhang, Q. Angew Chem Int Edit 2016, 55, 3334.

(17) Chen, X. M. H.; Ploux, O.; Liu, H. W. Biochemistry 1996, 35, 16412. 